ABSTRACTS COLLECTION

\title{
Abstracts from the 2021 Annual Scientific Meeting of the British and Irish Hypertension Society (BIHS)
}

(c) The Author(s), under exclusive licence to Springer Nature Limited 2021

Journal of Human Hypertension (2021) 35:1-19; https://doi.org/10.1038/s41371-021-00586-5

Hilton Brighton Metropole, Brighton, 13-15 September 2021

Conference Organizer: In Conference Ltd. on behalf of BIHS. https://bihsoc.org/events/.

All content was reviewed and approved by members of the British and Irish Hypertension Society, which held full responsibility for the abstract selections.

Sponsorship: Funding for the publication of this supplement was provided by the British and Irish Hypertension Society for the advancement of knowledge and dissemination of information concerning the pathophysiology, epidemiology, detection, investigation and treatment of arterial hypertension and related vascular diseases.

\section{0-1 Association of blood pressure with coronary microvascular dysfunction and cardiac dysfunction in asymptomatic type 2 diabetes}

Jian L. Yeo ${ }^{1,2^{*}}$, Gaurav S. Gulsin ${ }^{1,2}$, Emer M. Brady ${ }^{1,2}$, Lavanya Athithan ${ }^{1,2}$, Kelly S. Parke ${ }^{3}$, Gerry P. McCann ${ }^{1,2}$

${ }^{1}$ University of Leicester, Leicester, United Kingdom, ${ }^{2}$ NIHR Biomedical Research Centre, Leicester, United Kingdom, ${ }^{3}$ University Hospitals Leicester NHS Trust, Leicester, United Kingdom

Introduction: Hypertension is prevalent among individuals with type 2 diabetes (T2D) and contributes to heart failure events. We aimed to determine (1) the relationship between blood pressure (BP) and subclinical left ventricular (LV) dysfunction and perfusion in asymptomatic individuals with T2D, and (2) whether ambulatory $\mathrm{BP}$ is a better predictor of these abnormalities than office BP.

Methods: Asymptomatic people with T2D and no history of cardiovascular disease were prospectively enrolled and compared with age- and ethnicity-matched controls. Participants underwent clinical and metabolic profiling, echocardiography, and stress/rest cardiovascular magnetic resonance imaging with myocardial blood flow quantification. Office BP was calculated as the average of the final two of three readings at rest. Multivariable linear regression analysis was performed to assess the association of office and 24-h ambulatory BP with key cardiovascular outcome measures among those with T2D.

Results: 116 T2D (mean age $63.3 \pm 7.0$ years, 33\% female, $\mathrm{HbA} 1 \mathrm{c} 55.9 \pm 11.7 \mathrm{mmol} / \mathrm{mol}, 56 \%$ treated hypertension) and 38 controls (mean age $60.9 \pm 7.8$ years, $40 \%$ female, HbA1c $36.4 \pm$ $3.7 \mathrm{mmol} / \mathrm{mol}, 18 \%$ treated hypertension) were recruited with no significant differences in age, sex or ethnicity. Ambulatory but not office systolic BP $(130 \pm 12$ vs $124 \pm 13 \mathrm{mmHg}, p=0.007)$ was higher in T2D than controls. People with T2D had greater LV mass/ volume, systolic (lower global longitudinal strain) and diastolic (higher $\mathrm{E} / \mathrm{e}^{\prime}$ ratio) dysfunction and lower myocardial perfusion reserve $(2.8 \pm 0.8$ vs $3.1 \pm 0.8, p=0.03)$. After adjusting for clinical variables, systolic BP remained inversely associated with myocardial perfusion reserve $(r=-0.333, p<0.01)$ while diastolic BP was associated with impaired LV global longitudinal strain $(r=0.248, p<0.01)$. Ambulatory, but not office, systolic BP was independently associated with $\mathrm{E} / \mathrm{e}^{\prime}$.

Conclusions: Systolic BP is independently associated myocardial perfusion reserve and diastolic BP is independently associated with LV global longitudinal strain. These associations are consistent for both office and ambulatory BP.

Disclosures: GPM receives funding from NIHR through a Research Professorship award (RP-2017-08-ST2-007). GSG received funding from the British Heart Foundation through a Clinical Research Training Fellowship (FS/16/47/32190).

References: None.

\section{0-2 Omitted}

0-3 Safety and efficacy of cardioprotective medication reduction in older patients: a systematic review and metaanalysis

Rik Van Der Veen ${ }^{1 *}$, Lucy Goddard ${ }^{1}$, Joseph Lee ${ }^{1}$, Richard McManus ${ }^{1}$, Richard Hobbs ${ }^{1}$, Kamal Mahtani ${ }^{1}$, Constantinos Koshiaris ${ }^{1}$, James Sheppard ${ }^{1}$

${ }^{1}$ Nuffield Department of Primary Care Health Sciences, University of Oxford, Oxford, United Kingdom

Introduction: Polypharmacy is associated with an increased risk of harm and reduced quality of life (1). One solution is reducing cardioprotective medications in older adults to potentially reduce the risk of harm. However, there is currently no consensus on safety and efficacy of this approach (2). Therefore, we performed a systematic review of studies examining the efficacy of cardioprotective medication reduction in older patients. 
Methods: MEDLINE and Embase databases were searched from database inception to $16 / 06 / 2020$. Studies were eligible for inclusion if they were randomized controlled trials (RCTs) or nonrandomized studies examining the safety (e.g., change in blood pressure) and efficacy (e.g., quality of life) of cardioprotective medication reduction (anticoagulants, antihypertensives, antihyperlipidemic, or antiplatelets) in patients 75 years and over. Primary outcome was all-cause hospitalization. We pooled effects with random-effects meta-analyzes.

Results: We retrieved 8192 articles and included nine, four RCTs, and five non-randomized studies. Studies mean follow-up was 12 -weeks to 6.5 -years and covered 126,663 patients (range: 106-120,173) with a mean age of $75-84.4$ years. No association was detected between cardioprotective therapy reduction and allcause hospitalization (RR 1.15,95\% Cl 0.66-2.00; $n=955 ; 2$ studies; $I^{2}=0 \%$ ) or quality of life (SMD $-0.09,95 \% \mathrm{Cl}-0.22$ to 0.04 ; $n=955 ; 2$ studies; $I^{2}=0 \%$ ). Cardioprotective medication reduction was associated with an increase in all-cause mortality (RR 1.99, $95 \% \mathrm{Cl} 1.10-3.61 ; n=2950 ; 5$ studies; $l^{2}=57 \%$ ) and systolic blood pressure (MD $7.82 \mathrm{mmHg}, 95 \% \mathrm{Cl} 3.07-12.57 ; n=1262 ; 4$ studies; $\left.l^{2}=78 \%\right)$.

Conclusions: These findings suggest withdrawing cardioprotective therapy carries risks and should not be considered based on age alone. Overall, RCTs were few, and more appropriately powered trials are needed.

Disclosures: Drs. Sheppard, McManus and Hobbs were investigators on the OPTiMISE deprescribing trial

References:

1.Payne RA. The epidemiology of polypharmacy. Clin Med. 2016;16(5):465-9.

2.Krishnaswami A, Steinman MA, Goyal P, Zullo AR, Anderson TS, Birtcher KK, et al. Deprescribing in Older Adults With Cardiovascular Disease. J Am Coll Cardiol. 2019;73(20):2584-95.

\section{0-4 Omitted}

0-5 Air pollution and Blood Pressure change over time in $\mathbf{3 3 2 3}$ adolescents in London: differences by gender and ethnicity

\section{Alexis Karamanos*1, lan Mudway ${ }^{3}$, Andrew Webb², Yao Lu', Frank Kelly ${ }^{3}$, D. Dajnak ${ }^{3}$, S.D Beevers ${ }^{3}$, Christelle Elia ${ }^{1}$, Maria Maynard ${ }^{4}$, Seeromanie Harding ${ }^{1}$, J.K. Cruickshank ${ }^{1}$}

${ }^{1}$ Department of Nutritional Sciences, School of Life Course Sciences, Faculty of Life Sciences \& Medicine, King's College London, United Kingdom ${ }^{2}$ School of Cardiovascular Medicine \& Sciences King's College London British Heart Foundation Centre of Research Excellence, United Kingdom ${ }^{3}$ Environmental Research Group, MRC-PHE Centre for Environment and Health, School of Public Health, Faculty of Medicine, Imperial College London, United Kingdom ${ }^{4}$ School of Clinical and Applied Sciences, Leeds Beckett University, Leeds, United Kingdom

Background: There is little longitudinal evidence on how longerterm air pollution affects blood pressure (BP) development across adolescence.

Methods: Linear random effects modeling using 60 multiply imputed datasets was applied to multi-pollutant exposures and confounding variables (including change in weight, height, or waist, parental background, pubertal status, psychological measures, and activity) to explore how longer-term exposure to annual nitrogen dioxide $\left(\mathrm{NO}_{2}\right)$ and particulate matter $\left(\mathrm{PM}_{2.5}\right)$ based on adolescents' residential addresses, affected systolic/diastolic. Estimates are based on data from 3323 adolescents recruited in 5 ethnic groups from 51 schools in 10 London boroughs and followed up in the Determinants of Young Adult Social and Health "DASH" study between ages 11-13 and 14-16 years.

Results: During the 3-year follow up, mean $\pm \mathrm{SD}$ air $\mathrm{NO}_{2}$ was $41 \pm 4$, range $26.7-75.5, \mu \mathrm{g} / \mathrm{m}^{3}$, while similar $\mathrm{PM}_{2.5}$ values were $17.7 \pm 1.8,14.4-22.9, \mu \mathrm{g} / \mathrm{m}^{3}$. A $1-\mathrm{SD}$ increase in $\mathrm{NO}_{2}$ levels led to a SBP fall of $1.26(95 \% \mathrm{Cl} 0.80-1.71) \mathrm{mmHg}$ for girls and of 0.80 $(0.33-1.28) \mathrm{mmHg}$ for boys. A 1-SD PM 2.5 increase led to a 0.97 $(0.10-1.86) \mathrm{mmHg}$ increase in SBP for boys and a larger 2.37 $(1.51-3.23) \mathrm{mmHg}$ ) increase for girls. DBP effects were trivial. Ethnic specific effects were not evident.

Conclusion: From this first known British study of air pollution and adolescent $\mathrm{BP}$, higher air $\mathrm{NO}_{2}$ decreases $\mathrm{BP}$, especially in girls particularly while greater $\mathrm{PM}_{2.5}$ exposure increases BP. Whether these effects are due to time indoors, cooking gas exposure (1), and $\mathrm{PM}_{2.5}$ from wood-smoke fires remains unclear. Further longitudinal studies should clarify these contrasting effects in different socio-economic settings.

Disclosures: None.

Reference:

1. Webb AJ et al; Circulation research. 2020; https://www. ahajournals.org/doi/10.1161/CIRCRESAHA.120.316748.

0-6 Continuing versus stopping pre-stroke antihypertensive agents within $12 \mathrm{~h}$ of stroke onset is associated with worse outcomes after acute stroke

Jason Appleton ${ }^{1,2^{*}}$, Lisa Woodhouse ${ }^{1}$, Polly Scutt ${ }^{1}$, Lisa Everton ${ }^{1}$, Gwenllian Wilkinson ${ }^{1}$, Nikola Sprigg ${ }^{1,3}$, Philip Bath ${ }^{1,3}$

${ }^{1}$ Stroke Trials Unit, School of Medicine, University of Nottingham, Nottingham, United Kingdom, ${ }^{2}$ Stroke, University Hospitals Birmingham NHS Foundation Trust, Birmingham, United Kingdom, ${ }^{3}$ Stroke, Nottingham University Hospitals NHS Trust, Nottingham, United Kingdom

Introduction: It is unclear whether to continue or temporarily stop existing antihypertensive drugs in patients with acute stroke.

Methods: We performed a pre-specified subgroup analysis of patients enrolled into the Efficacy of Nitric Oxide in Stroke (ENOS) trial who were randomized to continue vs stop prior antihypertensive therapy within $12 \mathrm{~h}$ of stroke onset. The primary outcome was functional outcome, assessed with the modified Rankin Scale at 90 days by observers blinded to treatment assignment, and analyzed with ordinal logistic regression.

Results: Of 4011 patients, 2097 were randomized to continue vs stop prior antihypertensive treatment, and 384 (18.3\%, continue 185 , stop 199) were enrolled within $12 \mathrm{~h}$ of ictus: mean (SD) age 71.8 (11.8) years, female 193 (50.3\%), ischemic stroke $342(89.1 \%)$ and total anterior circulation syndrome $114(29.7 \%)$. As compared with stopping, continuing treatment within $12 \mathrm{~h}$ of onset lowered blood pressure by $15.5 / 9.6 \mathrm{mmHg}(p<0.001 /<0.001)$ by 7 days, shifted the modified Rankin Scale to a worse outcome by day 90 , adjusted common odds ratio (OR) $1.46(95 \% \mathrm{Cl} 1.01-2.11)$ and was associated with an increased death rate by day 90 (hazard ratio $2.17,95 \% \mathrm{Cl}$ 1.24-3.79). Other outcomes (disability-Barthel Index, quality of life -EQ-visual analog scale, cognition-telephone mini-mental state examination, and mood-Zung depression scale) were also worse with continuing treatment.

Conclusions: In this pre-specified subgroup analysis, continuing prior antihypertensive therapy within $12 \mathrm{~h}$ of stroke onset was unsafe with worse functional outcome, disability, cognition, mood, quality of life and increased death.

Disclosures: PMB is Stroke Association Professor of Stroke Medicine and a National Institute of Health Senior Investigator. The other authors have no disclosures. 
Clinical trial registration:

URL: http://www.isrctn.com/ISRCTN99414122. Unique Identifier: ISRCTN99414122.

References: None.

\section{0-7 Omitted}

0-8 Pre-load reduction augments the early phase of left ventricular contraction and improves diastolic function in hypertension

\section{Luca Faconti ${ }^{1 *}$, Bushra Farukh ${ }^{1}$, Ryan McNally ${ }^{1}$, Philip J Chowienczyk ${ }^{1}$}

${ }^{1}$ King's College London, Clinical Pharmacology, London, United Kingdom

Introduction: Increased cardiac pre-load has been implicated in the pathogenies of hypertension and the transition to heart failure (HF). Here we examined the effects of pre-load reduction on ventricular dynamics in clinically euvolemic subjects with hypertension and various degree of diastolic function.

Methods: Transthoracic echocardiography was used to assess superior vena cava (SVC) flow velocity (a measure of pre-load), systolic function including first-phase ejection fraction (EF1) and to estimate filling pressure/diastolic function (E/E' ratio). $E F 1$ is the ejection fraction up to the time of peak aortic blood flow and has been proposed as an index to study left ventricular systo-diastolic coupling [1]. Measurements were performed before and after lower-limb venous occlusion (LVO), a non-pharmacological intervention similar to lower-body negative pressure to cause a transient decrease in cardiac pre-load.

Results: In 136 hypertensive subjects (53\% male), LVO had minimal effects on blood pressure and heart rate but reduced SVC velocity by (mean $(95 \%$ confidence intervals)) $4.35(1.5,7.19) \mathrm{cm} / \mathrm{s}$ $(P=0.003)$. Conventional indices of left ventricular systolic function were unaffected but EF1 increased by $3.07(2.56,3.88) \%$ and $E / E^{\prime}$ decreased by $0.95(0.57,1.33)$; both $P<0.001$. In subjects with diastolic dysfunction/pre-clinical HF $(n=19)$ the increase in $E F 1$ and the decrease in $E / E^{\prime}$ were significantly greater compared to subjects with normal filling pressure. In subjects with pre-clinical HF, LVO increased EF1 from $18.22 \pm 4.73 \%$ to $22.91 \pm 6.89 \%$ and decrease $E / E^{\prime}$ from $15.02 \pm 4.75$ to $12.33 \pm 3.68$; $P<0.05$ for the comparison of $\triangle E F 1$ and $\triangle E / E^{\prime}$ between the two groups. The hemodynamic response to LVO was similar between subjects with pre-clinical HF and with normal filling pressure.

Conclusions: Acute reduction in pre-load by LVO augments the first phase of left ventricular ejection and improves diastolic function with effects that are greater in patients with diastolic dysfunction.

\section{Disclosures:}

No disclosures.

\section{Reference:}

[1] Gu H, Li Y, Fok H, Simpson J et al. Reduced first-phase ejection fraction and sustained myocardial wall stress in hypertensive patients with diastolic dysfunction: a manifestation of impaired shortening deactivation that links systolic to diastolic dysfunction and preserves systolic ejection. Hypertension. 2017;69:633-40.

\section{0-9 Arm based on LEg blood pressures (ABLE-BP): can leg blood pressure measurements predict brachial blood pressure? An individual participant data meta-analysis from the INTERPRESS-IPD Collaboration}

Sinead McDonagh ${ }^{1 *}$, James Sheppard ${ }^{2}$, Fiona Warren ${ }^{1}$, Kate Boddy ${ }^{1}$, Leon Farmer ${ }^{1}$, Helen Shore ${ }^{3}$, Phil Williams ${ }^{3}$, Philip
Lewis $^{4}$, Rachel Baumber ${ }^{5}$, A Jayne Fordham ${ }^{6}$, Una Martin7, Victor Aboyans ${ }^{8}$

${ }^{1}$ University of Exeter, Exeter, United Kingdom, ${ }^{2}$ University of Oxford, Oxford, United Kingdom, ${ }^{3}$ Thalidomide Trust, United Kingdom, ${ }^{4}$ Stockport NHS Foundation Trust, Stockport, United Kingdom, ${ }^{5}$ Royal National Orthopedic Hospital NHS Trust, Middlesex, United Kingdom, ${ }^{6}$ Mid Devon Medical Practice, Witheridge, United Kingdom, 7 University of Birmingham, Birmingham, United Kingdom, ${ }^{8}$ Dupuytren University Hospital, Limoges, France

Introduction: Hypertension is diagnosed and managed using blood pressures (BP) measured on the upper arm. Amputations, altered muscle tone after stroke or limb deformities can prevent accurate measurement of brachial BP. Leg BP measurement is advised as practical alternative, but limited data exist to guide clinicians' interpretation of leg BP values for hypertension diagnosis and treatment; therefore, we aimed to determine the relationship between arm and leg BP.

Methods: Individual participant data (IPD) met-analyzes using systolic arm and leg BP data from 14 studies within the INTERPRESS-IPD Collaboration were undertaken. We explored cross-sectional relationships between arm and leg BP using hierarchical linear regression with participants nested by study, in multivariable models. We predicted arm BP using leg BP quantified using area under receiver operating characteristic (AUROC) curves. Final models were validated using internal-external crossvalidation analysis.

Results: Arm and leg BP data were available for 33,710 individuals (mean age: 58.4 years, mean arm systolic/diastolic BP at baseline: $137.7 \mathrm{mmHg}, 44.7 \%$ female); $20.1 \%$ were smokers, 60.0 had hypertension, $14.6 \%$ had diabetes and $17.2 \%$ had cardiovascular disease. Mean leg systolic BP was $12.0 \mathrm{mmHg}(95 \% \mathrm{Cl}$, 8.8-15.2) higher than arm systolic BP. Descriptive modeling revealed systolic $B P$, female sex, smoking, total cholesterol, diabetes and ischemic heart disease were associated with reduced arm-leg systolic BP differences $(p<0.05)$. Age, body mass index and hypertension were associated with increased arm-leg systolic BP differences $(p<0.05)$. Derivation cohort AUROC curves for predicted arm BP from leg BP reduced from 0.93 to 0.89 with each $5 \mathrm{mmHg}$ reduction in BP from 160 to $130 \mathrm{mmHg}$.

Conclusions: This is the first IPD meta-analysis to describe the relationship between arm and leg systolic BP using an international cohort. Our findings will support clinicians and patients to use leg BP measurements in detecting and managing hypertension and cardiovascular risk more effectively.

Disclosures: The INTERPRESS-IPD Collaboration was established with a gran from the NIHR Research for Patient Benefit program (award number: PB-PG-0215-36009). The ABLE-BP project is supported by the Stroke Association (award number: SA PG 19/100043) and by the Thalidomide Trust (award number: $n / a)$.

\section{Reference:}

McDonagh STJ, Sheppard JP, Warren FC on behalf of the INTERPRESS-IPD Collaborators, et al. Arm Based on LEg blood pressures (ABLE-BP): can systolic leg blood pressure measurements predict systolic brachial blood pressure? Protocol for an individual participant data meta-analysis from the INTERPRESS-IPD collaboration. BMJ Open. 2021;11:e040481. https://doi.org/ 10.1136/bmjopen-2020-040481.

0-10 The relationship between serum sodium concentration and albuminuria: a retrospective cohort study 
Nicholas Cole ${ }^{1 *}$, Pauline Swift ${ }^{1}$, Rebecca Suckling ${ }^{1}$, Feng $\mathrm{He}^{2}$, Hugh Gallagher ${ }^{1}$, Jeremy van Vlymen ${ }^{3}$, Rachel Byford ${ }^{3}$, Simon de Lusignan ${ }^{3}$

${ }^{1}$ Epsom and St Helier NHS Trust, Carshalton, United Kingdom, ${ }^{2}$ Queen Mary University of London, London, United Kingdom, ${ }^{3}$ University of Oxford, Oxford, United Kingdom

Introduction: Randomized-controlled trials demonstrate that lowering dietary salt intake reduces albuminuria, an early marker of renal damage and a sensitive predictor of adverse cardiovascular outcomes (1-3). The mechanisms underlying this effect are uncertain but small differences in serum sodium concentration may be important $(4,5)$. This retrospective cohort study investigated the hypothesis that higher serum sodium concentration is a risk factor for albuminuria (defined as a urine albumin:creatinine ratio, or UACR, $\geq 3 \mathrm{mg} / \mathrm{mmol}$ ).

Methods: Primary care data from the Royal College of General Practitioners Research and Surveillance Center was used to identify 47,294 individuals with a UACR result available between April 2010 and March 2015, and no known albuminuria prior to this. Exclusion criteria were: missing or abnormal serum sodium concentration at baseline ( $<135$ or $>146 \mathrm{mmol} / \mathrm{L})$; age $<18$ years; diabetes mellitus; decompensated liver disease; heart failure; and stage 5 chronic kidney disease.

Results: Over five years, albuminuria was identified in 8329 $(17.7 \%)$ of the study cohort. There was a significant "U-shaped" relationship between serum sodium concentration and UACR, after adjustment for known risk factors. There was no association between serum sodium concentration and blood pressure.

Conclusions: The finding of a positive association between higher serum sodium concentration and albuminuria is in support of the hypothesis, but the inverse relationship between serum sodium concentration and albuminuria at lower concentrations warrants further explanation.

Disclosures: FJH is a member of Consensus Action on Salt and Health (CASH) and World Action on Salt \& Health (WASH). Both $\mathrm{CASH}$ and WASH are non-profit charitable organizations and $\mathrm{FJH}$ does not receive any financial support from CASH or WASH. The remaining authors declare no competing interests.

\section{References:}

1. McMahon EJ, et al. J Am Soc Nephrol. 2013; 24: 2096-103.

2. Chronic Kidney Disease Prognosis Consortium, et al. Lancet. 2010; 375: 2073-18

3. Gansevoort RT, et al. Kidney Int. 2011; 80: 93-104.

4. de Wardener $\mathrm{HE}$, et al. Plasma sodium and hypertension. Kidney Int. 2004; 66: 2454-66.

5. He FJ, et al. Plasma sodium: ignored and underestimated. Hypertension. 2005; 45: 98-102.

0-11 Central pulse pressure in adolescence is more strongly associated with future left ventricle mass than brachial pulse pressure

Chloe Park ${ }^{1 *}$, Siana Jones ${ }^{1}$, Laura Howe ${ }^{2}$, Diana Ferreira ${ }^{2}$, Abigail Fraser', Debbie Lawlor ${ }^{2}$, John Deanfield', Suzanne Williams ${ }^{1}$, Hannah Taylor ${ }^{1}$, Alicja Rapala ${ }^{1}$, Nish Chaturvedi ${ }^{1}$, Alun Hughes ${ }^{1}$

${ }^{1}$ MRC Unit for Lifelong Health and Ageing at UCL, London, United Kingdom, ${ }^{2}$ University of Bristol, Bristol, United Kingdom

Introduction: Left ventricle mass (LVM) is an indicator of target organ damage and a predictor of cardiovascular disease independent of blood pressure. Cross-sectional investigation in adolescence has shown that central pulse pressure (cPP) is more closely associated with LVM than brachial pulse pressure (bPP) (1). Longitudinal follow-up of these adolescents is required to determine if cPP or bPP is more closely associated with future LVM.

Methods: 1259 participants (777 female) in the Avon Longitudinal Study of Parents and Children (ALSPAC) underwent repeat measurements of bPP and CPP (Vicorder), and echocardiography aged 17 years and 24 years. LVM was calculated and indexed to height $^{1.7}$ (LVMI). Multivariable linear regression was used to assess longitudinal associations between CPP/bPP aged 17 years and LVMI aged 24 years. Data for sexes were pooled and adjusted for age, sex and parental socioeconomic position (model 1). Bootstrapping (10,000 replications) was used to compare CPP and bPP associations.

Results: Aged 17 years, the difference between bPP and CPP was marked (mean difference $(95 \% \mathrm{Cl})=20.7(20.5,21.0) \mathrm{mmHg}$ ). CPP and bPP were both positively associated with future LVMI aged 24 years $\left(B(95 \mathrm{Cl})\right.$ per $1 \mathrm{mmHg}$ increase in CPP $0.29 \mathrm{~g} / \mathrm{m}^{1.7}$ $(0.24,0.35) p<0.0001$ and bPP $\left.0.18 \mathrm{~g} / \mathrm{m}^{1.7}(0.15,0.22) p<0.0001\right)$ but associations were stronger for cPP (bootstrap $p<0.0001$ ). Differences in strength of associations remained after adjustment (model 1) ( $(95 \mathrm{Cl}) \mathrm{CPP} 0.40 \mathrm{~g} / \mathrm{m}^{1.7}(0.29,0.51) p<0.0001$, bPP $\left.0.26 \mathrm{~g} / \mathrm{m}^{1.7}(0.18,0.34) p<0.0001\right)$.

Conclusions: Adolescent CPP is more closely associated with future LVM than bPP. These results suggest that central rather than brachial blood pressure may be a better measure of future risk in adolescence

\section{Disclosures: None.}

Reference:

1. Ferreira DLS, Fraser A, Howe LD, Jones S, Smith GD, Lawlor DA, et al. Associations of central and peripheral blood pressure with cardiac structure and function in an adolescent birth cohort: the Avon longitudinal study of parents and children. J Am Coll Cardiol. 2015;65(18):2048-50.

\section{0-12 Omitted}

\section{0-13 Omitted}

0-14 Regular paracetamol use and blood pressure in people with hypertension: the PATH-BP trial

Iain M. Maclntyre ${ }^{1 *}$, Emma J. Turtle ${ }^{2}$, Tariq E. Farrah ${ }^{2}$, Catriona Graham $^{2}$, James W. Dear', David J. Webb ${ }^{2}$

${ }^{1}$ NHS Lothian, Edinburgh, United Kingdom, ${ }^{2}$ The Centre for Cardiovascular Science, University of Edinburgh, Edinburgh, United Kingdom

Introduction: Paracetamol is widely used as first-line therapy for chronic pain due to its perceived safety and the assumption that, unlike non-steroidal anti-inflammatory drugs, it has little or no effect on blood pressure (BP). Although observational studies suggest that paracetamol may increase BP [1], clinical trials are lacking. We therefore studied the effects of regular paracetamol dosing on BP in individuals with hypertension.

Methods: In this double-blind, placebo-controlled, crossover study, 110 hypertensive individuals (both treated and untreated) were randomized to receive paracetamol $1 \mathrm{~g}$ four times daily or matched placebo for 2 weeks followed by a 2 -week washout period before crossing over to the alternate treatment. 24-h ambulatory BP was measured at the beginning and end of each treatment period. The primary outcome was a comparison of the 
change in mean daytime systolic BP from baseline to end of treatment between placebo and paracetamol arms.

Results: 103 patients completed both arms of the study. Regular paracetamol, compared to placebo, resulted in a significant increase in mean daytime systolic BP $(132.8 \pm 10.5$ to $136.5 \pm$ $10.1 \mathrm{mmHg}$ vs $133.9 \pm 10.3$ to $132.5 \pm 9.9, p<0.0001)$ with a placebo-corrected increase of $4.7 \mathrm{mmHg}(95 \%$ confidence interval [CI] 2.9-6.6) and mean daytime diastolic BP $(81.2 \pm 8.0$ to $82.1 \pm 7.8$ vs $81.7 \pm 7.9$ to $80.9 \pm 7.8 \mathrm{mmHg}, p=0.005)$ with a placebocorrected increase of $1.6 \mathrm{mmHg}(95 \% \mathrm{Cl} 0.5-2.7)$. Similar findings were seen for 24-h ambulatory and clinic BP.

Conclusions: Regular paracetamol intake of $4 \mathrm{~g}$ daily increases systolic BP in individuals with hypertension by around $5 \mathrm{mmHg}$ when compared to placebo, increasing cardiovascular risk and calling into question the safety of regular paracetamol use in this situation.

Disclosures: This study was funded by a British Heart Foundation grant (PG/13/26/3012 8).

\section{Reference:}

1. Turtle EJ, Dear JW, Webb DJ. A systematic review of the effect of paracetamol on blood pressure in hypertensive and nonhypertensive subjects. Brit J Clin Pharmacol. 2013;75:1396-405.

\section{P-1 Aldosterone-producing adenomas with dual mutations of GNA11/Q and CTNNB1 lead to hypertension in puberty, pregnancy or menopause, and complete cure by adrenalectomy}

\author{
Giulia Argentesi ${ }^{1,2^{*}}$, Junhua Zhou ${ }^{1,2}$, Elena Azizan ${ }^{3}$, Claudia P \\ Cabrera $^{4}$, Emily Cottrell ${ }^{5}$, Xilin Wu ${ }^{1,2}$, Emily Goodchild ${ }^{1,2}$, \\ Roger Foo', Chaz Mein ${ }^{6}$, Eva Wozniak ${ }^{6}$, William Drake ${ }^{1,2}$, \\ Helen Storr ${ }^{5}$, Morris Brown ${ }^{1,2}$
}

${ }^{1}$ Endocrine Hypertension, Department of Clinical Pharmacology, William Harvey Research Institute, Queen Mary University of London, London, United Kingdom, ${ }^{2} \mathrm{NIHR}$ Barts Cardiovascular Biomedical Research Centre, Barts and The London School of Medicine and Dentistry, Queen Mary University of London, United Kingdom, ${ }^{3}$ Department of Medicine, The National University of Malaysia (UKM) Medical Centre, Malaysia, ${ }^{4}$ Centre for Translational Bioinformatics, William Harvey Research Institute, Queen Mary University of London, United Kingdom, ${ }^{5}$ Centre for Endocrinology, William Harvey Research Institute, Queen Mary University of London, United Kingdom, ${ }^{6}$ Barts and London Genome Centre, School of Medicine and Dentistry, Blizard Institute, Queen Mary University of London, United kingdom, ${ }^{7}$ Cardiovascular Research Institute, Yong Loo Lin School of Medicine, National University of Singapore, Singapore

Introduction: Primary aldosteronism (PA) is the cause of high-risk hypertension in $5-10 \%$ of unselected patients. Guidelines predict cure rate to be $30-60 \%$ post adrenalectomy [1]. Whole exome sequencing found somatic non-ion channel mutations of CTNNB1 within 3 patients all seemingly caused by the LH/HCG surge of pregnancy, puberty or menopause [2]. LHCG receptors are known to be present within the adrenal cortex and play a role in steroidogenesis. LH-dependent cortisol and androgen secreting adenomas of the adrenal have been described $[3,4]$ Our aim was to uncover further PA patients at time of LH/HCG surge and explore their geno-phenotype.

Methods: 10 patients were identified, of whom 9 presented either in the first trimester of pregnancy, puberty or menopause. Data was collected of blood pressures, renin, aldosterone and potassium levels. Post adrenalectomy tissue was procured with patient consent. DNA and RNA were extracted for genotyping and gene expression. Transfection studies were performed on primary adrenal cells and cell lines.
Results: Average blood pressure pre-surgery: 172.7/ $102.6 \mathrm{mmHg}$ and post-surgery: $116.8 / 67.3 \mathrm{mmHg}$. Pre-surgery aldosterone: $1485.9 \mathrm{pmol} / \mathrm{L}$ and post-surgery: $219 \mathrm{pmol} / \mathrm{L}$. Presurgery potassium: $2.8 \mathrm{mmol} / \mathrm{L}$ and post-surgery: $4.7 \mathrm{mmol} / \mathrm{L}$. All patients were off medications post-surgery. On sanger sequencing, all had CTNNB1 mutations which varied between S45F and P, G34E and R, T41A and S33C. We discovered a unique somatic mutation of the G-protein coupled receptor, GNA11/Q, at the residue, Q209, p.GIn209His/Pro or Leu, required for GTPase activity. 9 double mutant adenomas had an $18.09(P<0.0001)$ fold higher $L H C G R$ expression compared to adenomas with wild type or adjacent tissue. Aldosterone/ CYP11B2 was significantly higher in transfections with both mutations $(P<0.0005)$.

Conclusions: We have demonstrated a unique cohort of patients presenting with explosive onset of PA at times of high LH/HCG surge. All achieved clinical and biochemical cure post adrenalectomy, the elevated aldosterone likely having a transitory effect upon blood pressure dysregulation.

Disclosures: Please note data is part of a larger study in press in Nature Genetics.

\section{References:}

1. Funder, J.W., et al., The management of primary aldosteronism: case detection, diagnosis, and treatment: an endocrine society clinical practice guideline. J Clin Endocrinol Metab. 2016. 101(5): 1889-916.

2. Teo, A.E., et al., Pregnancy, primary aldosteronism, and adrenal CTNNB1 mutations. N Engl J Med. 2015. 373(15): 1429-36.

3. Plockinger, U., et al., Functional implications of $\mathrm{LH} / \mathrm{hCG}$ receptors in pregnancy-induced cushing syndrome. J Endocr Soc. 2017. 1(1): 57-71.

4. Leinonen, P., et al., Testosterone-secreting virilizing adrenal adenoma with human chorionic gonadotrophin receptors and 21hydroxylase deficiency. Clin Endocrinol. 1991. 34(1): 31-5.

\section{P-2 Kidney allograft function and late post-transplant hypertension: time-dependent effect analysis}

\section{Ekamol Tantisattamo ${ }^{{ }^{*}}$, Sakditad Saowapa ${ }^{2}$, Natchaya Polpichai $^{3}$, Natsuki Eguchi ${ }^{1}$}

${ }^{1}$ Harold Simmons Center for Kidney Disease Research and Epidemiology, Division of Nephrology, Hypertension and Kidney Transplantation, Department of Medicine, University of California Irvine School of Medicine, Orange, United States, ${ }^{2}$ Faculty of Medicine Ramathibodi Hospital, Mahidol University, Bangkok, Thailand, ${ }^{3}$ Faculty of Medicine Songklanagarin Hospital, Prince of Songkla University, Songkhla, Thailand

Introduction: Blood pressure changes at different post-kidney transplant periods. The study aims to determine the association between kidney allograft function and hypertension over several time points during post-transplant periods.

Methods: A single-center retrospective cohort study including kidney transplant recipients, who received transplantation between 2012 and 2015, was conducted to examine the association between estimated glomerular filtration rate (eGFR) at 12 weeks post-transplant (as arbitrary baseline allograft function) and hypertension by multivariable Cox regression analysis with a time-dependent effect at 12, 24, 36, and 48 weeks posttransplantation. Systolic and diastolic hypertension (SHTN, DHTN) was defined as SBP $\geq 130$ and DBP $\geq 80 \mathrm{mmHg}$, respectively.

Results: Of all 105 patients, the mean age \pm SD was $54 \pm 12$ and $61 \%$ was female. Mean eGFR at a 12 -week post-transplant was $56 \pm 18 \mathrm{ml} / \mathrm{min} / 1.73 \mathrm{~m}^{2}$. The majority of patients $(58 \%)$ had stage 3 CKD with a mean eGFR of $46 \pm 8 \mathrm{ml} / \mathrm{min} / 1.73 \mathrm{~m}^{2}$. For every period post-transplant at 12,24 , and 36 weeks, there was no 
association between eGFR at a 12-week post-transplant and both SHTN and DHTN. However, at 48 weeks post-transplant, every $1 \mathrm{ml} / \mathrm{min} / 1.73 \mathrm{~m}^{2}$ increase in eGFR was significantly associated with a $2.06 \%$ decreased risk of SHTN (HR 0.9798, $p 0.010,95 \% \mathrm{Cl}$ $0.9647,0.9951)$. After adjusted for age, gender, type of transplant, and pre-transplant body mass index, an increase in eGFR at 12 weeks post-transplant was associated with a $1.78 \%$ lowered risk of SHTN at 48 weeks post-transplant (HR 0.9825342, p 0.038, 95\% $\mathrm{Cl} 0.9663,0.9991)$. The eGFR at a 12 -week post-transplant was not associated with DHTN at a 48-week post-transplant both unadjusted and adjusted analysis.

Conclusions: Allograft function is associated with late posttransplant SHTN, but not with the early SHTN or DHTN at all posttransplant periods. Allograft function may be affected by immunological and non-immunological factors at different periods posttransplant, which subsequently lead to late post-transplant SHTN.

Disclosures: The authors declared no competing interests.

Reference:

1. Tantisattamo et al. Approach and management of hypertension after kidney transplantation. Front Med. 2020; 7:229. https://doi.org/10.3389/fmed.2020.00229.

\section{P-3 The effect of anti-hypertensive treatment on exercise blood pressure and exercise capacity in older adults}

\section{Siana Jones ${ }^{1 *}$, Martin Schultz ${ }^{2}$, Chloe Park ${ }^{1}$, Therese Tillin ${ }^{1}$, Nishi Chaturvedi ${ }^{1}$, Alun Hughes ${ }^{1}$}

${ }^{1}$ MRC Unit for Lifelong Health \& Ageing at University College London, London, United Kingdom, ${ }^{2}$ Menzies Institute for Medical Research, University of Tasmania, Hobart, Australia

Introduction: An exaggerated blood pressure (BP) response to exercise is a risk factor for cardiovascular disease independent of resting BP.[1-3] The effect of pharmacological treatment on exercise BP and exercise capacity in older adults is largely unknown. This study investigates these effects in a populationbased sample of older adults.

Methods: Participants enrolled in the Southall and Brent Revisited (SABRE) study attended a research clinic and undertook a 6-min stepper test with expired gas analysis to assess self-paced exercise capacity ( $\mathrm{VO}_{2}$ achieved). BP was measured at 2-min intervals. Participants were stratified into groups by treatment status and resting BP control: normotensive, treated/controlled, treated/uncontrolled and untreated/uncontrolled. Exercise capacity and highest exercise systolic and diastolic BP (exSBP \& exDBP) were compared between groups using potential outcome means $(95 \% \mathrm{Cls})$ calculated from treatment effects estimated using augmented inverse probability weighted propensity scores. Estimates were adjusted for confounders and were considered with and without inclusion of participants prescribed $\beta$-blockers.

Results: 670 participants were included (age \pm SD: $73 \pm 6.6$ years, $56 \%$ male, 58\% treated). ExSBP was similar in normotensive versus treated/controlled individuals $(180(175,186) \mathrm{mmHg}$ vs 179 $(175,184) \mathrm{mmHg}, p=0.779)$ but higher in treated/uncontrolled and untreated/uncontrolled groups $(194(190,199) \mathrm{mmHg}, p<$ $0.001 ; 197(192,203) \mathrm{mmHg}, p<0.001)$. ExDBP was similar in normotensive versus treated/controlled and treated/uncontrolled groups $(92(88,95) \mathrm{mmHg}$ vs $89(87,91) \mathrm{mmHg}, p=0.250$ and $91(89$, 93) $\mathrm{mmHg}, p=0.766$ ) but was higher in the untreated/uncontrolled group $(98(95,102) \mathrm{mmHg}, p=0.009)$. $\dot{\mathrm{VO}}_{2}$ achieved was lower in treated groups (normotensive: $16.7(16.0,17.4) \mathrm{ml} / \mathrm{min} / \mathrm{kg}$ vs treated/controlled: $15.5(14.9,16.1) \mathrm{ml} / \mathrm{min} / \mathrm{kg}, \quad p=0.011$ and treated/uncontrolled: $15.1(14.9,16.1) \mathrm{ml} / \mathrm{min} / \mathrm{kg}, p<0.001)$ but similar between normotensive and the untreated/uncontrolled group $(17.0(16.2,17.9) \mathrm{ml} / \mathrm{min} / \mathrm{kg}, p=0.547)$. Similar results were observed when participants prescribed $\beta$-blockers were excluded.

Conclusions: Anti-hypertensive treatment lowered exDBP to levels observed in normotensive older adults but only lowered exSBP if resting BP was also controlled. Compared to normotensive older adults, exercise capacity is reduced in treated/controlled and treated/uncontrolled groups, but similar in untreated/ uncontrolled older adults.

Disclosures: The authors declared no competing interests.

References:

1. Schultz, M.G., A. La Gerche, J.E. Sharman. Blood pressure response to exercise and cardiovascular disease. Curr Hypertens Rep. 2017. 19(11): p. 89.

2. Schultz, M.G., et al. Exercise-induced hypertension, cardiovascular events, and mortality in patients undergoing exercise stress testing: a systematic review and meta-analysis. Am J Hypertens. 2013. 26(3): 357-66.

3. Thanassoulis, G., et al. Relations of exercise blood pressure response to cardiovascular risk factors and vascular function in the Framingham Heart Study. Circulation, 2012. 125(23): 2836-43.

P-4 Association between 24-h urinary sodium concentration and body mass index in children with primary hypertension

\section{Cheentan Singh ${ }^{1 *}$, Haotian $\mathrm{Gu}^{2}$, Joanne Newton ${ }^{1}$, Manish Sinha ${ }^{1,2}$}

${ }^{1}$ Evelina London Children's Hospital, Gstt, London, United Kingdom, ${ }^{2}$ Cardiovascular Division, Kings College London, London, United Kingdom

Introduction: In children and young people (C\&YP) referred for evaluation of primary hypertension $(\mathrm{PH})$, to (i) report on 24-hour urine sodium concentrations; and (ii) evaluate its relationship with blood pressure, excess weight and left ventricular mass.

Methods: Prospective data including C\&YP over a recent 6 year period (2013-2018) with new diagnosis of Primary hypertension were included. Diagnosis of hypertension was confirmed on 24-h ABPM. Body mass index (BMI), 24-h sodium concentration (24 h$\mathrm{Na}$ ) and indexed LV mass (LVMi) from echocardiography were measured.

Results: Eighty-four children with PH [male: 45 (53.6\%), mean age $14.2 \pm 2.5$ years] were included. Forty three patients $(66.1 \%)$ had $24 \mathrm{~h}-\mathrm{Na}$ above $100 \mathrm{mmol} /$ day. The average BMI of $26.7 \pm$ $7.3 \mathrm{~kg} / \mathrm{m}^{2}$ (BMl $z$ score $1.32 \pm 0.97$ ), 24 -h systolic BP of $128 \pm$ $10 \mathrm{mmHg}$ (SPB $z$ score $1.56 \pm 1.59$ ), 24-h diastolic BP of $73 \pm$ $8 \mathrm{mmHg}$ (DBP $z$ score $0.98 \pm 1.49$ ), $24-\mathrm{h}$ MAP of $91 \pm 7 \mathrm{mmHg}$ (MAP $z$ score $1.46 \pm 1.45)$, LVMi of $38.0 \pm 14.2 \mathrm{~g} / \mathrm{m}^{2.7}$ and $24 \mathrm{~h}-\mathrm{Na}$ of $124.2 \pm 70.4 \mathrm{mmol} / \mathrm{s}$.

In a multivariate Linear regression analysis model, BMI $z$ score was positively associated with $24 \mathrm{~h}-\mathrm{Na}(\beta=0.364, p=0.024)$ independent of age, 24-h systolic blood pressure and LVMi.

Conclusions: These data highlight the clinical utility of measurement of $24 \mathrm{~h}-\mathrm{Na}$ provides a "personalized" evaluation to explain the need for dietary modification to C\&YP and their families. The independent association in those with excess BMI highlight importance of salt intake reduction in all obese patients with $\mathrm{PH}$.

Disclosures: None.

Reference:

Hanevold, C.D. Sodium intake and blood pressure in children. Curr Hypertens Rep. 2013;15:417-25. https://doi.org/10.1007/ s11906-013-0382-z. 
P-5 COVID-19 blood pressure endothelium interaction study (OBELIX)—blood pressure assessment, vascular phenotyping and RAAS-fingerprinting of COVID-19 survivors

Linsay McCallum ${ }^{1}$, Stefanie Lip ${ }^{1 *}$, Francisco Rios ${ }^{1}$, Karla Neves $^{1}$, Jason Kilmartin ${ }^{2}$, Eleanor Murray', Salil Reetoo', Laura Knox ${ }^{2}$, Maggie Rostron ${ }^{2}$, Angela K. Lucas-Herald ${ }^{1}$, Clea Du Toit ${ }^{1}$, Tom Guzik ${ }^{1}$, Christian Delles ${ }^{1}$, Augusto C. Montezano ${ }^{1}$, Dame Anna F. Dominiczak', Sandosh Padmanabhan ${ }^{1}$, Rhian M. Touyz ${ }^{1}$

${ }^{1}$ University of Glasgow, Glasgow, United Kingdom, ${ }^{2}$ Queen Elizabeth University Hospital, Glasgow G51 4TF, United Kingdom

Introduction: Longer-term cardiovascular consequences of COVID-19 are unquantified however the potential for residual heart and vascular inflammation with perturbation of the reninangiotensin-aldosterone system (RAAS) in the months post-illness may represent a nidus for new-onset hypertension and cardiovascular disease. This study aims to investigate if recovered COVID-19 patients are at greater risk of hypertension and RAAS alterations in the medium-longer term.

Methods: Patients aged 30-60 years, admitted with suspected/ confirmed COVID-19, during 01/04/2020-31/05/2020, were eligible. COVID-19 case definition: positive SARS-CoV-2 RT-PCR and/or diagnostic chest $x$-ray; control: negative SARS-CoV-2 RT-PCR, negative SARS-CoV-2 lgG antibody and non-diagnostic chest $x$-ray. Patients with a history of hypertension or on blood pressure (BP) lowering drugs were excluded. Participants had 24-hour ambulatory BP monitoring (ABPM), vascular phenotyping and RAASfingerprinting at $\geq 12$ weeks post discharge. Primary outcome was the ABPM 24-h systolic blood pressure (SBP). Multivariable logistic regression was used to assess differences between groups.

\section{Results:}

30 SARS-CoV-2 positive and 18 negative participants were recruited. The SARS-CoV-2 positive group were older (51[7.3] vs 45 [9.1]; $p=0.018)$ with lower discharge SBP (120.5[10.1], vs 128.3 [14.6]; $p=0.063)$. The SARS-CoV-2 positive group had an $8.6 \mathrm{mmHg}$ higher ABPM 24-h SBP (8.6[0.99-16.3]; $p=0.028)$, a $9.4 \mathrm{mmHg}$ higher ABPM day SBP $(9.4[1.5-17.3] ; p=0.021)$ and $a$ $4.6 \mathrm{mmHg}$ higher ABPM day DBP (4.5[0.1-9.1]; $p=0.048)$. Office SBP measured at the study visit was $11.5 \mathrm{mmHg}$ higher in the SARS-CoV-2 positive group (11.5[3.1-19.8]; $p=0.008)$. Renin and Angiotensin-1-10 levels were lower in the SARS-CoV-2 positive group $(-0.39[-0.85$ to 0.06$] ; p=0.08 ;-0.65[-1.20$ to -0.09$] ; p=$ 0.023 respectively).

Conclusions: SARS-CoV-2 positive participants, without history of hypertension, had significantly elevated 24-h and daytime SBP at $\geq 12$ weeks post-hospital discharge. This was associated with reduced renin and Angiotensin-1-10 levels. Together, our findings indicate that COVID-19 survivors may be at risk of developing hypertension, which appears to be a low-renin form.

Disclosures: None.

References: None.

\section{P-6 Withdrawn}

P-7 Familial hyperkalaemic hypertension is associated with immunodeficiency
Barian Mohidin ${ }^{1 *}$, Rhys Evans ${ }^{1}$, Elizabeth Wan ${ }^{1}$, Keith Siew ${ }^{1}$, Stephen B Walsh'

${ }^{1}$ Department of Renal Medicine, UCL, London, United Kingdom

Introduction: Familial hyperkalaemic hypertension (FHHt) is also known as Gordon syndrome or pseudohypoaldosteronism type II. It is an autosomal dominant condition that leads to salt retention through over activation of the sodium chloride co-transporter (NCC) in the distal convoluted tubule causing salt-sensitive hypertension. Pathogenic mutations in WNK1, WNK4, CUL3 and KLHL3 cause FHHt. Our recent study on patients with salt-losing tubulopathies (SLTs e.g., Gitelman syndrome) found significantly altered immunity, predisposing patients to a variety of infections ${ }^{1}$. We studied whether FHHt (a salt retaining tubulopathy) also led to an altered immune phenotype.

Methods: 12 patients (6 female, median age 38 years) with genetically confirmed FHHt (8 KLHL3, 2 WNK1, 2 WNK4) were administered a validated questionnaire ${ }^{1}$. The study was ethically approved (REC 05/Q0508/6) and patients gave informed consent. Data collected included past medical history, medications, autoimmunity, atopy, allergy status, childhood infections and recurrent adult infections. This was compared to 24 healthy controls and 47 patients with SLT, using the Mann Whitney or Wilcoxon signed rank test as appropriate.

Results: Patients with FHHt were found to have significantly more viral $(p=0.0094)$ and fungal infections $(p=0.04)$ compared to healthy controls. They also experienced more recurrent upper respiratory tract infections $(p=0.04)$ and urinary tract infections $(p=0.04)$. Compared to patients with SLT, FHHt patients suffered significantly more recurrent viral infections $(p=0.0021)$ but less allergic disease $(p=0.03)$.

Conclusions: Our data suggests altered immunity in patients with $\mathrm{FHHt}$. Their phenotype and range of infections were different to both healthy controls and patients with SLT. These data may imply that there may be an immune phenotype in hypertensive patients with a high salt intake. This, the molecular mechanism in $\mathrm{FHHt}$ patients and whether the phenotype is amenable to treatment requires further investigation.

Disclosures: None.

Reference:

1 Evans RDR et al. Nat Commun. 2020; 11: 4368.

P-8 Demographic characteristics of childhood hypertension in the United Kingdom

\section{Emily Haseler $^{{ }^{*}}$, Cheentan Singh ${ }^{2}$, Joanna Newton ${ }^{3}$, Nabil Melhem $^{3}$, Manish Sinha ${ }^{3,4}$}

${ }^{1}$ University Hospital Lewisham, London, United Kingdom, ${ }^{2}$ North Middlesex University Hospital, London, United Kingdom, ${ }^{3}$ Evelina London Children's Hospital, London, United Kingdom, ${ }^{4}$ King's College London, London, United Kingdom

Introduction: We report on demographic characteristics in Children and Young People (C\&YP) from the largest dedicated pediatric hypertension clinic in the UK.

Methods: Prospective data including all C\&YP over a recent 6 year period (2013-2018). Patients categorized as normotensive, white coat hypertension (WCH), primary hypertension $(\mathrm{PH})$ and secondary hypertension ( $\mathrm{SH}$ ) and compared across age groups $<6$ $(n=162), 6$ to $<12(n=114)$, and 12 to $<18$ years $(n=272)$.

Results: 548 C\&YP [58.2\% girls] with median age of 11.9 (IQR 10.8) years. 232 (42.3\%) were normotensive; of those hypertensive 
$(n=316), 46(15 \%), 83(26 \%)$ and 187 (59\%) were WCH, PH and SH respectively. There was a bimodal age distribution for those with $\mathrm{SH}$ with peaks in $<6$ and adolescent years, whereas $\mathrm{PH}$ and $\mathrm{WCH}$ peaked solely in adolescence.

Children <6years significantly more likely to have normotension following evaluation using appropriate out-of-office monitoring. If hypertensive, they were significantly more likely to have $\mathrm{SH}$ and less likely to have body mass index $>95$ percentile compared with older children. In those aged <6years, prevalence of $\mathrm{WCH}$ and $\mathrm{PH}$ both was $<0.5 \%$ and significantly lower than older age groups.

There were lower numbers of hypertensive children of nonWhite Ethnicity $<6$ years. Amongst those with hypertension, those of non-White Ethnicity had higher prevalence of PH but comparable rates of $\mathrm{WCH}$ when compared with White patients.

Children of normal weight had higher prevalence of non-White ethnicity with $\mathrm{PH}$. Amongst those with excess weight ( $>85$ th percentile), significantly more older children but no difference in distribution of hypertension categories by ethnicity [ $p=0.07]$.

Conclusions: Hypertensive young children are most likely to have secondary hypertension and negligible rates of $\mathrm{WCH}$ and $\mathrm{PH}$. Primary hypertension including WCH accounts for $40 \%$ of hypertension in C\&YP in the UK with the highest prevalence in adolescence, non-White Ethnicity and excess weight.

Disclosures: None.

References: None.

\section{P-9 Drug-related problems among hypertensive and heart failure patients on the medical wards of a teaching hospital in Ghana}

Mark Amankwa Harrison ${ }^{1 *}$, Afia Frimpomaa Asare Marfo², Daniel Nii Amoo Ankrah ${ }^{1}$, Dorcas Poku Boateng ${ }^{1}$, Florence Amah Nkansah ${ }^{1}$, Frempomaa Nelson ${ }^{1}$, Augustine Annan ${ }^{1}$, Grace Owusu-Aboagye', Franklin Acheampong', Kwame Ohene Buabeng ${ }^{2}$

${ }^{1}$ Pharmacy Department, Korle Bu Teaching Hospital, Accra, Ghana, ${ }^{2}$ Department of Pharmacy Practice, Kwame Nkrumah University of Science and Technology, Kumasi, Ghana

Introduction: Drug-related problems (DRPs) lead to substantial morbidity, mortality and costs to health systems, but pharmacists' interventions can prevent these consequences. The aim of this study was to determine the frequency and characteristics of drugrelated problems, their associated factors, and pharmacists' interventions.

Methods: It was a cross sectional study of hypertensive and heart failure (HF) patients with DRPs admitted to the medical wards of the Korle Bu teaching hospital in Ghana. Data collection was over a 12-week period. Patients' drug therapies were reviewed by clinical pharmacists using the hospital's structured pharmaceutical care form. Patients' medical information, DRPs and pharmaceutical care interventions provided were collected. DRPs and interventions were classified using an adapted Pharmaceutical Care Network Europe (PCNE) classification tool.

Results: A total of 247 DRPs were identified in 134 hypertensive and HF patients (hypertensive $(n)=126 ; \mathrm{HF}(n)=56$ ). The average frequency of DRPs was $1.84 /$ patient $(S D=1.039)$. The most prevalent DRPs were counseling need (43\%), untreated indication (20\%), monitoring need (17.8\%) and drug-drug interactions (17.8\%). Most frequently implicated drugs in DRPs were anticoagulants (48.1\%), anti-hypertensive drugs (23.3\%) and antibiotics (22.6\%). Number of drugs (total) prescribed was positively associated significantly with number of DRPs $(B=0.212 ; p$-value $=0.024)$. Number of anti-hypertensive and HF drugs were both significantly associated with number of DRPs (p.008; $p=0.001)$. There were 235 interventions (average of 1.75/patient). Intervention acceptance at prescriber level was $71.6 \%$. The majority (70.6\%) of interventions accepted were implemented.

Conclusions: Drug-related problems were frequent among patients on anticoagulants, anti-hypertensive drugs and antibiotics. DRPs were positively associated with number of prescribed drugs. Many hypertensive and heart failure patients need medication counseling, and review of their drug therapy by clinical pharmacists can improve care.

Disclosures: None.

References:

Allemann, Samuel S. et al. Pharmaceutical care: the PCNE definition 2013. Int J Clin Pharm. 2014;36.3: 544-55.

\section{P-10 Cardiovascular pharmacotherapy and COVID-19}

Stefanie Lip ${ }^{1 *}$, Linsay McCallum ${ }^{1}$, Clea Du Toit ${ }^{1}$, Jason Kilmartin ${ }^{2}$, Eleanor Murray ${ }^{1}$, Salil Reetoo ${ }^{1}$, Laura Knox, Maggie Rostron $^{2}$, Tomasz Guzik ${ }^{2}$, Christian Delles ${ }^{1}$, Dame Anna Dominiczak $^{2}$, Rhian Touyz ${ }^{2}$, Sandosh Padmanabhan ${ }^{2}$

${ }^{1}$ University of Glasgow, Glasgow, United Kingdom, ${ }^{2}$ Queen Elizabeth University Hospital, Glasgow, United Kingdom

Introduction: COVID-19 is associated with cardiovascular vulnerabilities at all stages of infection (pre-infection, acute phase and subsequent chronic phase). The major cardiometabolic drivers identified to have epidemiological and mechanistic associations with COVID-19 are abnormal adiposity, dysglycaemia, dyslipidaemia, and hypertension. Recent findings demonstrate that cardiovascular risk reduction interventions may have short-term benefits on COVID-19 risk and outcomes. Our aim was to investigate the effect of cardiovascular pharmacotherapy on SARS-CoV-2 infection.

Methods: Data on hospital admissions, RT-PCR tests, prescriptions and investigations were obtained through record linkage for all patients admitted between 01/04/2020 and 31/05/2020 to NHS Greater Glasgow \& Clyde hospitals. The relationship between cardiovascular pharmacotherapy (antihypertensives and statins) and SARS-CoV-2 infection was analyzed using multivariable logistic regression models.

Results: From 218,472 patient records 3610 patients had confirmed COVID-19 (clinical criteria or a positive RT-PCR test) and 18,050 propensity matched controls were selected. The average age was 62 years, $55 \%$ females, $43 \%$ resided in the most deprived areas (SIMD:1), 46\% were taking antihypertensives and 35\% were on statins. In the multivariable logistic regression model, the risk of SARS-CoV-2 infection was greater in those with cancer, pulmonary or renal disease. Those on antihypertensive or statin therapy had an $11 \%$ (O.R.[95\% Cl] -0.89 [0.82-0.97], $p=0.007)$ and $9 \%(0.91$ [0.83-0.99], $p=0.03$ ) lower risk of SARS-CoV-2 infection, respectively. The protective effect of statins and antihypertensive drugs were apparent only in those on both agents $(0.82$ [0.74-0.92], $p=$ 0.001).

Conclusions: Patients on prior treatment with statins and antihypertensive drugs have a reduced risk of SARS-CoV-2 infection. The observed beneficial effect of statins and antihypertensive drugs warrants further investigation to address critical gaps in prevention, and acute/chronic care of patients with COVID-19.

Disclosures: None.

References: None. 
P-11 Is frailty a useful measure to inform management of hypertension in older people? A qualitative study exploring the patient's perspective

\section{Oliver Todd ${ }^{1}{ }^{*}$, Rebecca Hawkins ${ }^{1}$, James P Sheppard ${ }^{2}$, Richard J McManus ${ }^{2}$, Kenneth Rockwood ${ }^{3}$, Chris P Gale ${ }^{4,5}$, Marlous Hall $^{4,5}$, Andrew Clegg ${ }^{1}$, Mary Godfrey ${ }^{1}$}

${ }^{1}$ University Of Leeds, Bradford, United Kingdom, ${ }^{2}$ Nuffield Department of Primary Care Health Sciences, University of Oxford, Oxford, United Kingdom, ${ }^{3}$ Geriatric Medicine Research, Dalhousie University, Halifax, Canada, ${ }^{4}$ Leeds Institute of Cardiovascular and Metabolic Medicine, Leeds, United Kingdom, ${ }^{5}$ Leeds Institute for Data Analytics, University of Leeds, University of Leeds, United Kingdom

Introduction: In the management of hypertension, UK NICE guidelines recommend using clinical judgement when making treatment decisions in older people with frailty [1]. We explored the understanding of older people with hypertension regarding frailty and it might affect their care.

Methods: Semi-structured qualitative interview study with ten older people. Participants were recruited from the Community Ageing Research 75+ (CARE75+) cohort study [2], had hypertension and frailty. They were purposively sampled for variation in gender and age. Each participant was interviewed twice, a week apart, at the participant's own home and used biographical methods. Analysis was inductive, working from a reflexive stance, and focused on the content and form of stories told.

Results: Participants age ranged from 77 to 93 and half were women. Seven lived alone, two with their spouses, one with family. Hypertension had been diagnosed: during pregnancy (2); following a stroke; following a fall (1); or, on screening (3). Three themes emerged in the narratives. Firstly, ageing was more visible in others than in oneself. Most did not identify as frail and described the term in negative and value-laden terms. Secondly, how a person conceived frailty offered a means to understand that person on their terms: how they identified themselves, what they valued and what they feared. Thirdly, hypertension seemed abstract, medicalised with decision making delegated to doctors. A minority seemed involved in any sharing of decision making with respect to the management of their hypertension.

Conclusions: We found that the perception of frailty among patients themselves may differ from that of a clinician. Understanding how a patient conceives of frailty may represent a means to understand what they value, so as to better engage patients in shared decision-making regarding BP-lowering treatment.

Disclosures: None.

References:

[1] National Institute for Health and Care Excellence, Hypertension in adults: diagnosis and management. N. Clinical Guideline [CG136] 2019.

[2] Heaven A, Brown L, Young J, Teale E, Hawkins R, Spilsbury K, Mountain G, Young T, Goodwin V, Hanratty B, Chew-Graham C, Brundle C, Mahmood F, Jacob I, Daffu-O'Reilly A, Clegg A. Community ageing research $75+$ study (CARE75+): an experimental ageing and frailty research cohort. BMJ Open. 2019, 9(3), e026744.

P-12 Longitudinal trajectories of pulse wave velocity and cognition in the Irish longitudinal study of ageing (TILDA)

David Moloney ${ }^{1 *}$, Rose Anne Kenny ${ }^{1}$, Roman Romero-Ortuno ${ }^{1}$

${ }^{1}$ Falls and Syncope Unit, St James's Hospital, Dublin 8, Ireland

Introduction: Arterial stiffness is a marker of vascular ageing and carotid-femoral pulse wave velocity (cf-PWV) is a standard measurement method. A raised cf-PWV in individuals has been associated cross-sectionally with poor cognition and longitudinally with increased incidence of hypertension, cardiovascular events, and mortality. We hypothesized that individuals who have accelerated vascular ageing will also have greater cognitive decline and explored that potential association in Wave 1 (W1) and Wave 3 (W3) of The Irish Longitudinal Study on Ageing (TILDA).

Methods: cf-PWV was measured by tonometry $\left(\right.$ Vicorder $\left.^{\circledR}\right)$ and the average of two PWV measurements between the carotid and femoral arteries was used. Global cognition was assessed at the health center, using the Mini Mental State Exam (MMSE) and Montreal Cognitive Assessment (MOCA), which were repeated at W1 (4-year follow-up period). Linear regression analyzes were employed to predict change in cognitive measure by PWV change quintiles, whilst controlling by age, sex, education, smoking, body mass index, cardiovascular diseases and depression.

Results: 3274 participants had data at both W1 and W3 for analysis (mean age 64 , mean cf-PWV at W1 $=10.4 \mathrm{~m} / \mathrm{s}$ ). In the fully adjusted model, the highest quintile of cf-PWV change was significantly associated with greater decreases in MMSE $(0.303,95 \%$ Cl: $0.054-0.397, p=0.011)$ and MOCA $(0.416,95 \% \mathrm{Cl}: 0.185-0.726$, $p=0.027$ ) between $\mathrm{W} 1$ and $\mathrm{W} 3$.

Conclusions: Over a 4-year period, participants in the highest quintile of cf-PWV increase demonstrated greater decreases in both MOCA and MMSE when compared to those in the lowest quintile. Treating causes of accelerated cf-PWV progression may have a role in preserving global cognition.

Disclosures: None.

References: None.

P-13 The additional prognostic value of arterial stiffness for primary cardiovascular events in pre and stage-1 hypertensives

Holly Pavey ${ }^{1}{ }^{*}$, Ian Wilkinson ${ }^{1}$, Kaisa Maki-Petaja ${ }^{1}$, Yoav BenShlomo ${ }^{2}$, Angela Wood ${ }^{1}$, Carmel McEniery ${ }^{1}$

${ }^{1}$ University of Cambridge, Cambridge, United Kingdom, ${ }^{2}$ University of Bristol, Bristol, United Kingdom

Introduction: Aortic stiffness is a novel cardiovascular (CV) risk factor. A previous meta-analysis demonstrated that aortic pulse wave velocity (aPWV, a robust measure of aortic stiffness) added value beyond traditional risk factors when predicting future CV events (1). However, the majority of individuals were older or at high CV risk, whereas the predictive value of aPWV in middle-risk individuals, in whom more accurate risk prediction is likely to have the greatest benefit, is unknown.

Methods: We analyzed aPWV and outcome data from 13,541 middle-risk individuals (pre- and stage- 1 hypertensives, without a history of CV disease) from 11 population-based cohorts. Cox proportional hazard models were fitted and study-specific hazard ratios pooled in a 2 -stage random-effects meta-analysis. A novel risk score including aPWV was derived and validated using bootstrap resampling methods and compared to established CVD risk scores such as the Framingham CVD risk equation. Performance measures included measures of discrimination, calibration as well as retrospective and prospective net reclassification (NRI) indices.

Results: A 1 standard deviation (SD) increase in log(aPWV) was associated with a $1.21(95 \% \mathrm{Cl}: 1.11,1.34)$ fold increase of CV events. Compared with traditional risk factors, addition of aPWV significantly improved the predictive ability of the model (C-statistic: $0.0038(0.0002,0.0075), p=0.04)$. This improvement was greater than the inclusion of total cholesterol to Framingham CVD risk factors: $0.0036(0.0006,0.0066), p=0.02$. A validated novel CVD risk equation showed good model performance and 
calibration. The novel risk model improved reclassification of individuals by almost 5\% (prospective NRI: 4.99 (95\% Cl: 3.87, 6.10)), compared to the Framingham CVD equation, even after study-specific recalibration.

Conclusions: Addition of aPWV to traditional CV risk factors improves prediction and classification of primary CV events in individuals at middle-risk of CV events. Assessing aortic stiffness is likely to be useful in general health screening.

\section{Disclosures: None.}

\section{Reference:}

1. Yoav B-S, Melissa S, Chris B, Margaret M, Simon GA, Emelia JB, Pierre $B$ et al. Aortic pulse wave velocity improves cardiovascular event prediction: an individual participant meta-analysis of prospective observational data from 17,635 subjects. J Am Coll Cardiol. 2014;63(7):636-46.

\section{P-14 Does frailty modify the association between systolic blood pressure and major adverse cardiovascular or cerebrovascular outcomes? Retrospective analysis using Welsh routine data from the secure anonymised information linkage (SAIL) Databank}

Oliver Todd ${ }^{1 *}$, Chris Wilkinson $^{2}$, Joe Hollinghurst ${ }^{3}$, Ashley Akbari $^{3}$, James P Sheppard ${ }^{4}$, Richard McManus ${ }^{4}$, Ronan A Lyons $^{3}$, Kenneth Rockwood ${ }^{5}$, Chris P Gale ${ }^{6,7}$, Marlous Hall ${ }^{6,7}$, Andrew Clegg ${ }^{1}$

${ }^{1}$ Academic Unit for Ageing and Stroke Research, University Of Leeds, Bradford, United Kingdom, ${ }^{2}$ Population Health Sciences Institute, Faculty of Medical Sciences, Newcastle University, Newcastle, United Kingdom, ${ }^{3}$ Health Data Research UK, Swansea University, Swansea, United Kingdom, ${ }^{4}$ Nuffield Department of Primary Care Health Sciences, University of Oxford, Oxford, United Kingdom, ${ }^{5}$ Geriatric Medicine Research, Dalhousie University, Halifax, Canada, ${ }^{6}$ Leeds Institute of Cardiovascular and Metabolic Medicine, University of Leeds, Leeds, United Kingdom, ' Leeds Institute for Data Analytics, University of Leeds, Leeds, United Kingdom

Introduction: The target level of systolic blood pressure (sBP) at which antihypertensive treatment provides overall benefit remains the subject of debate for older people with hypertension. This study investigated whether frailty can identify a population of older people for whom sBP-associated risk of cardiovascular disease is different.

Methods: Retrospective cohort study using population-level linked primary and secondary care electronic health record (EHR) data from the Welsh Secure Anonymised Information Linkage (SAIL) Databank. Participants were aged 65 years and over and had a diagnosis of hypertension but no history of established cardiovascular disease. We extracted: lowestsBP at first attendance to primary care in 2007; BP-lowering treatment; cardiovascular risk (QRISK-3); and frailty (electronic frailty index). Time-to-event analysis measured first major adverse cardiovascular or cerebrovascular event (MACCE) including stroke, myocardial infarction, heart failure or cardiovascular death through 10-year follow-up.

Results: The analytic cohort included 145,598 patients with a mean age of 75 years (SD 7). After adjustment for QRISK-3 and treatment, the relative risk of MACCE was increased at sBP $<120 \mathrm{~mm} \mathrm{Hg}$ and $>180 \mathrm{~mm} \mathrm{Hg}$ in people who were robust or had mild frailty. However, in people with moderate or severe frailty, there was no difference in relative risk of MACCE according to SBP. There was no evidence that the association between $S B P$ and MACCE was modified by frailty $(p=0.419)$. However, there was a significant interaction between frailty and antihypertensive treatment in predicting future MACCE events $(p<0.001)$.

Conclusions: Our study found no evidence that the associations between SBP and MACCE varied according to a patient's frailty status, but there was evidence of that the association of BPlowering treatment and MACCE varied according to frailty status. Disclosures: None.

References: None.

\section{P-15 OMITTED}

\section{P-16 Tissue Doppler $E^{\prime}$ velocity and $E / E^{\prime}$ predict 19-year} cardiovascular mortality in hypertension

Anenta Ratneswaren ${ }^{1 *}$, Anoop SV Shah ${ }^{1}$, Simon AM Thom ${ }^{1}$, Andrew SP Sharp ${ }^{1}$, Darrel P Francis ${ }^{1}$, Alice V Stanton ${ }^{1}$, Neil R Poulter ${ }^{1}$, Peter S Sever ${ }^{1}$, Alun D Hughes ${ }^{1}$, Jamil Mayet ${ }^{1}$

${ }^{1}$ Imperial College London, London, United Kingdom

Introduction: We tested whether tissue Doppler assessment of LV diastolic function predicts cardiovascular (CV) mortality in the Hypertension Associated Cardiovascular Disease sub-study of the Anglo-Scandinavian Cardiac Outcomes Trial (ASCOT).

Methods: This study comprised the 519 patients recruited to the St Mary's Hospital ASCOT site who were followed for 19 years with mortality flagged by the Office for National Statistics. We used deaths reported on or before 31st January 2019. CV deaths include those due to coronary heart disease, stroke and other CV causes.

Echocardiography was performed one year after BP control. Mean tissue Doppler $E^{\prime}$ was calculated as the average of septal, lateral and inferior wall measurements over three cycles. The ratio of transmitral Doppler E wave velocity and composite mean of $E^{\prime}$ was used to calculate $E / E^{\prime}$ ratio.

Results: After a median of 19 years 317 patients survived (mean age at baseline 60.7 years, 38 female patients) and 202 did not (mean age at baseline 68.1 years, 30 female patients), with 23 CHD deaths, 11 stroke deaths and 27 deaths due to other CV causes.

Unadjusted analysis showed a strong association between CV mortality and $E^{\prime}(\mathrm{HR}=0.74, p<0.005)$ and $E / E^{\prime}(\mathrm{HR}=1.18, p<$ $0.005)$. The association between $C V$ mortality and $E^{\prime}$ persisted after adjustment for age and sex ( $\mathrm{HR}=0.83, p=0.02)$ and adjustment for age, sex and systolic $\mathrm{BP}(\mathrm{HR}=0.83, p=0.03)$.

The association between CV mortality and $E / E^{\prime}$ also persisted after adjustment for age and $\operatorname{sex}(H R=1.12, p=0.01)$ and adjustment for age, sex and systolic $\mathrm{BP}(\mathrm{HR}=1.11, p=0.04)$.

Conclusions: Tissue Doppler $E^{\prime}$ velocity and $E / E^{\prime}$ predicts $19-$ year CV mortality in a hypertensive population independent of age, sex and systolic BP.

Disclosures: None.

References:

1. Sharp AS, et al. Tissue Doppler $E / E^{\prime}$ ratio is a powerful predictor of primary cardiac events in a hypertensive population: an ASCOT substudy. Eur Heart J. 2010; Mar 31(6): 747-52

2. Sever PS, et al. Rationale, design, methods and baseline demography of participants of the anglo-scandinavian cardiac outcomes trial. ASCOT investigators. J Hypertens. 2001; Jun 19(6): 1139-47

3. Stanton A, et al. An intensive phenotyping study to enable the future examination of genetic influences on hypertensionassociated cardiovascular disease. J Hum Hypertens. 2001; Aug 15 Suppl 1: S13-8

P-17 Which arm to measure blood pressure: does the higher or lower reading arm best reflect systolic blood pressure? An 
individual participant data meta-analysis from the INTERPRESS-IPD Collaboration

\section{Christopher Clark $^{1 *}$, Fiona Warren ${ }^{1}$, Kate Boddy ${ }^{1}$, Sinead McDonagh ${ }^{1}$, Sarah Moore ${ }^{1}$, Lyne Cloutier ${ }^{2}$, Rod Taylor ${ }^{3}$, Angela Shore', Richard McManus ${ }^{4}$, Victor Aboyans ${ }^{5}$, John Campbell ${ }^{1}$}

${ }^{1}$ University of Exeter Medical School, Exeter, United Kingdom, ${ }^{2}$ Université du Québec à Trois-Rivières, Trois-Rivières, Canada, ${ }^{3}$ University of Glasgow, Glasgow, United Kingdom, ${ }^{4}$ University of Oxford, Oxford, United Kingdom, ${ }^{5}$ Dupuytren University Hospital, Limoges, France

Introduction: Hypertension guidelines recommend measuring blood pressure (BP) in both arms, adopting the higher arm readings for diagnosis and management. To our knowledge, no publications justify this recommendation. Using data from 23 cohorts in the INTERPRESS-IPD Collaboration, we examined the impacts of using higher or lower arms systolic BPs on estimated all-cause mortality, cardiovascular mortality and cardiovascular event risks.

Methods: Individual participant data meta-analyzes. We examined associations between higher and lower reading arm BPs and event outcomes using multivariable Cox regression models stratified by study. Models using higher and lower arm BPs were compared using Akaike's information criteria, Harrell's C-statistics and likelihood ratios. Prediction of events based on Framingham, Atherosclerotic Cardiovascular Disease (ASCVD) and Systematic COronary Risk Evaluation (SCORE) risk scores calculated from either higher or lower arm BPs were compared. Proportions of participants reclassified across guideline recommended BP and cardiovascular risk treatment thresholds were also explored.

Results: Bilateral BP measurements existed for 53,172 participants: mean age 60 years; 48\% female. Higher arm BP better predicted all-cause mortality, cardiovascular mortality, and cardiovascular events compared to lower arm BP after adjusting for age, sex, smoking, ethnicity, total cholesterol, hypertension and diabetes $(P<0.001$ all outcomes). For participants without cardiovascular disease, higher arm BP better predicted cardiovascular events using Framingham $(N=23,278)$ or ASCVD $(N=18,557)$ risk scores compared to lower arm BP $(P<0.001$ for both). Switching from lower arm to higher arm BP reclassified $4.6 \%, 3.5 \%$ and $7.7 \%$ of participants to a higher guideline-recommended risk category using Framingham, ASCVD and SCORE respectively; $12 \%$ were reclassified from below to above $130 \mathrm{mmHg}$ and $140 \mathrm{mmHg}$ systolic thresholds $(P<0.001$ for all).

Conclusions: Higher reading arm systolic BPs better predict outcomes in comparison to the lower arm. Both arms should be measured during assessment and the higher arm BP adopted for diagnosis and management of hypertension.

Disclosures: The INTERPRESS-IPD Collaboration was funded by the National institute for Health Research (NIHR) Research for Patient Benefit Program (PB-PG-0215-36009). RMcM receives support from the NIHR Oxford CLAHRC. ACS receives support from the NIHR Exeter Clinical Research Facility. The views expressed are those of the authors and not necessarily those of the NIHR, the NHS or the Department of Health and Social Care.

References: None.

P-18 Aortic distensibility predicts adverse cardiovascular events in the UK biobank cohort

Marina Cecelja ${ }^{1 *}$, Esther Puyol ${ }^{1}$, Bram Ruijsink ${ }^{1}$, Harriet Godwin ${ }^{1}$, Andrew King ${ }^{1}$, Reza Razavi ${ }^{1}$, Phil Chowienczyk ${ }^{1}$

${ }^{1}$ King's College London, London, United Kingdom
Introduction: Our aim was to investigate the association of aortic distensibility, pulse pressure (PP) and arterial stiffness index (SI) with cardiovascular risk factors and adverse cardiovascular events in participants from the UK Biobank cohort.

Methods: Aortic distensibility was measured from cardiovascular magnetic resonance images using automated analysis in 8435 participants from the UK Biobank cohort. The associations of aortic distensibility, brachial PP and SI (obtained by finger photoplethysmography) with conventional risk factors was examined by multivariate regression and with incident cardiovascular events by Cox proportional-hazards regression.

Results: Mean $( \pm S D)$ values of aortic distensibility for men and women were $1.77 \pm 1.15$ and $2.10 \pm 1.45 \quad(P<0.0001) \quad 10^{-3}$ $\mathrm{mmHg}^{-1}$, respectively. In multivariable regression analysis, aortic distensibility associated with age, mean arterial pressure, heart rate, weight, and plasma glucose but not male gender, cholesterol or current smoking. During an average follow-up of $2.8 \pm 1.3$ years, 92 participants experienced a cardiovascular event and 6 died. Higher aortic distensibility was associated with reduced risk of cardiovascular events (adjusted hazard ratio 0.61; 95\% confidence interval $(\mathrm{Cl})$ : $0.41-0.91, P=0.016)$. There was no evidence of an association between PP and risk of cardiovascular events (adjusted hazard ratio $1.00 ; 95 \% \mathrm{Cl}: 0.98-1.02, P=0.715)$ or $\mathrm{SI}$ and cardiovascular events (adjusted hazard ratio 1.02; 95\% Cl: 0.95-1.10, $P=$ 0.535).

Conclusions: Aortic distensibility, but not PP or SI, significantly predicted new-onset cardiovascular events. These findings suggest that aortic distensibility, when compared to PP and SI, is a more sensitive measure of cardiovascular ageing that relates more strongly to adverse cardiovascular outcome.

Disclosures: None.

References: None.

\section{P-19 Omitted}

\section{P-20 Omitted}

P-21 Associations between statins and adverse events in primary prevention of cardiovascular disease: a systematic review with meta-analysis

Ting Cai ${ }^{1 *}$, Lucy Abel ${ }^{1}$, Oliver Langford ${ }^{2}$, Genevieve Monaghan ${ }^{1}$, Jeffrey K Aronson ${ }^{1}$, Richard J Stevens ${ }^{1}$, Sarah Lay-Flurrie ${ }^{1}$, Constantinos Koshiaris ${ }^{1}$, Richard J McManus ${ }^{1}$, FD Richard Hobbs ${ }^{1}$, James P Sheppard ${ }^{1}$

${ }^{1}$ Nuffield Department of Primary Care Health Sciences, University of Oxford, Oxford, United Kingdom, ${ }^{2}$ Alzheimer's Therapeutic Research Institute, University of Southern California, Los Angeles, United States

Introduction: Concerns about adverse events pose a dilemma for the use of statins on patients without established cardiovascular diseases (CVD). Better understanding of the associations between statins and specific adverse events in this population is needed to support clinical decisions on statin treatment for primary prevention of CVD.

Methods: We conducted a systematic review of randomized controlled trials in adults without a history of CVD that compared statins with non-statin controls and reported any of the following adverse events: self-reported muscle symptoms, clinicallyconfirmed muscle disorders, liver dysfunction, renal insufficiency, diabetes, and eye conditions. We also examined three major cardiovascular events, including myocardial infarction, stroke, and 
death from CVD, as secondary outcomes. Pair-wise meta-analysis was conducted to calculate odds ratio (OR) and absolute risk difference (ARD) with 95\% confidence interval (Cl) for each outcome.

Results: We included 35 studies with 101,548 participants followed-up for an average of 4.2 years. Statins were associated with an increased risk of self-reported muscle symptoms (20 trials, $\mathrm{OR}=1.06$ [95\%Cl: $1.00-1.12], \mathrm{ARD}=13$ [95\%Cl: 1-28] per 10,000 person-years), liver dysfunction (21 trials, $\mathrm{OR}=1.33$ [1.12-1.58], $\mathrm{ARD}=8$ [3-14] per 10,000 person-years), and eye conditions (5 trials, $\mathrm{OR}=1.22[1.03-1.46], \mathrm{ARD}=14[2-28]$ per 10,000 personyears). These risk increases did not exceed the risk reductions of major cardiovascular events (35 fewer cases per 10,000 personyears) by statins in the included studies. No significant associations were found between statins and clinically-confirmed muscle disorders or diabetes in these studies.

Conclusions: The risk of adverse events attributable to statins in patients without previous CVD was low and did not outweigh the efficacy of statins in reducing CVD, suggesting that the benefit to harm balance of statins in primary prevention of CVD is favorable.

Disclosures: None.

References: None.

\section{P-22 Effect of diuretics on plasma aldosterone in primary hypertension: a systematic review and meta-analysis}

\section{Ryan McNally ${ }^{1 *}$, Luca Faconti ${ }^{1}$, Bushra Farukh ${ }^{1}$, Phil Chowienczyk ${ }^{1}$}

\section{${ }^{1}$ King's College London, London, United Kingdom}

Introduction: The renin-angiotensin-aldosterone system (RAAS) plays a major role in cardiovascular homeostasis and plasma aldosterone (PA) is a well-established mediator of cardiac and vascular remodeling. Whether effects of diuretics on PA differ according to class of diuretic or according to changes in potassium is unknown. Here we perform a systematic review and metaanalysis of randomized-controlled trials investigating the effects of diuretic therapy on PA.

Methods: The review was carried out in accordance with PRISMA guidelines (1). Three databases were searched: MEDLINE, EMBASE and CENTRAL up to and including 5 October 2020. Studies were eligible if they were a randomized-controlled trial examining antihypertensive effects of either a thiazide, thiazidelike, loop, mineralocorticoid receptor antagonist (MRA) or potassium-sparing diuretic with a duration of at least 1 week. All studies were required to have examined PA with results available before and during diuretic treatment or during treatment with placebo. Titles were firstly screened by title and abstract for relevancy before full-text articles were assessed for eligibility. A random-effects model was used to compensate for between-study heterogeneity with calculation of the standardized mean difference in PA and its $95 \%$ confidence interval $(\mathrm{Cl})$.

Results: A total of 1139 articles were retrieved of which 45 met the pre-specified inclusion/exclusion criteria. The average standardized difference in mean PA change was similar for all classes of diuretic (mean, 95\% Cl); thiazide/thiazide-like $0.304(0.169$, $0.440)$, loop $0.927(0.37,1.49)$, MRA/potassium-sparing 0.264 $(0.174,0.355)$ and combination $0.466(0.142,0.789), Q=6.475, P=$ 0.091 , but with a tendency for the effects of the loop and combination diuretics to be greater. There was no significant association between change in PA and change in serum potassium.

Conclusions: In RCTs of diuretic therapy in hypertension, there is an increase in PA with all classes of diuretic and no significant between-class heterogeneity.

Disclosures: None.

\section{Reference:}

1. Moher D, Liberati A, Tetzlaff J, Altman DG. Preferred reporting items for systematic reviews and meta-analyzes: the PRISMA statement. J Clin Epidemiol. 2009;62(10):1006-12.

P-23 Factors associated with blood pressure measured using home blood pressure monitors in the TIME (treatment in morning versus evening) study

\section{Keeran Vickneson ${ }^{1 *}$, Amy Rogers ${ }^{1}$, David Rorie ${ }^{1}$, Thomas M MacDonald ${ }^{1}$, Isla S Mackenzie ${ }^{1}$}

\section{${ }^{1}$ University of Dundee, Dundee, United Kingdom}

Introduction: Home-monitoring of blood pressure is increasingly used. Previous studies by our group have identified several factors, including smoking and obesity, that are negatively associated with home blood pressure monitor (HBPM) ownership and usage among participants in the TIME study. This analysis aims to identify whether these factors are associated with blood pressure in a large cohort of individuals with hypertension.

Methods: The TIME study is a remote decentralized randomized trial investigating the effect of day versus night-time dosing of antihypertensive medication on cardiovascular outcomes in 21,104 participants with hypertension (1). Participants with HBPMs were invited to submit BP measurements every 3 months via the TIME study website. Multivariable linear regressions were constructed to determine factors associated with baseline systolic (SBP) and diastolic blood pressure (DBP).

Results: 12,152 participants provided a baseline BP measurement. 5205 (42.8\%) reported mean BP below 135/85 mmHg. Age was positively associated with SBP $(+0.14 \mathrm{mmHg}$ per year, $p<0.001)$ but negatively associated with DBP $(-0.26 \mathrm{mmHg}$ per year, $p<0.001)$. The number of antihypertensive medications taken was associated negatively with DBP $(-0.47 \mathrm{mmHg}$ per additional agent, $p<0.001)$. Participants with a hypertensive family history had higher SBP and DBP $(+0.75 \mathrm{mmHg}$ and $+0.58 \mathrm{mmHg}, p<0.01)$. Current smoking and BMI were both associated with higher SBP $(+3.27 \mathrm{mmHg}, p<0.05 ;+0.16 \mathrm{mmHg}$ per $\left.\mathrm{kg} / \mathrm{m}^{2}, \quad p<0.001\right)$ and DBP $(+1.04 \mathrm{mmHg}, \quad p<0.001$; $+0.11 \mathrm{mmHg}$ per $\mathrm{kg} / \mathrm{m}^{2}, p<0.001$, respectively). Ex-smokers had higher systolic $(+0.52 \mathrm{mmHg}, p<0.05)$ but lower diastolic pressures $(-0.64 \mathrm{mmHg}, p<0.001)$ as compared with non-smokers. Participants with diabetes or myocardial infarction (MI) had lower SBP $(-0.94 \mathrm{mmHg}, p<0.05$ and $-2.72 \mathrm{mmHg}, p<0.001)$. Diabetes, $\mathrm{MI}$, stroke, and impaired kidney function were all associated with lower DBP $(p<0.01)$. After 12 months $(n=3428)$, mean BP dropped from $132.1 / 76.7 \mathrm{mmHg}$ to $131.0 / 76.1 \mathrm{mmHg}(p<0.001)$.

Conclusion: This study provides insights into factors associated with blood pressure in participants in a remote hypertension study who are prescribed at least one antihypertensive medication.

Disclosures: The TIME study is funded by the British Heart Foundation and supported by the British and Irish Hypertension Society.

\section{Reference}

1. Rorie DA, et al. BMJ Open. 2016;6(2):e010313. https://doi.org/ 10.1136/bmjopen-2015-010313.

\section{P-24 Omitted}

P-25 Obese hypertensive patients-is it time to consider new treatment paradigms? 
Cormac Kennedy ${ }^{1}$, Richard Farnan ${ }^{1}$, John Stinson ${ }^{1}$, Mary Hall $^{2}$, Linda Hemeryck ${ }^{2}$, Patricia O'Connor ${ }^{2}$, Martina Hennessy', Michael Barry ${ }^{1}$

${ }^{1}$ Trinity College Dublin, Dublin, Ireland, ${ }^{2}$ St James Hospital, Dublin, Ireland

Introduction: Recently glucagon-like peptide-1 (GLP-1) analogs have demonstrated a reduction in blood pressure when used to treat obesity [1]. Is there a need to reconsider the treatment paradigm for obese hypertensive patients to incorporate antiobesity agents? We aimed to determine if obese hypertensive patients attending our specialist hypertension clinic are adequately treated by elucidating their anti-hypertensive treatment and blood pressure (BP) control when stratified by body mass index (BMI) category.

Methods: Consent for inclusion in the study was requested from patients attending our clinic over a three-month period. Clinical details were recorded from patient charts to characterize. The NICE target BP of $<140 / 90 \mathrm{mmHg}$ was used to decipher BP control. Those with resistant hypertension were identified by their BP control and number of medications. The study was approved by our hospital research ethics committee.

Results: 170 patients were recruited for the study and had anthropometric measurements. Their mean age was 49.5 years and BMl $30.3 \mathrm{~kg} / \mathrm{m}^{2}$. $50 \%$ of the cohort were obese, while $10 \%$ were morbidly obese. The obese patients were treated with more anti-hypertensive medications compared to their non-obese hypertensive counterparts ( 2.1 vs $1.6 ; p=0.009)$ and the mean number of anti-hypertensives increased with each incremental BMI category. Despite more treatment, less BP control was evident as $\mathrm{BMI}$ category increased. Consistent with these results, resistant hypertension was more prevalent in obese patients $\left(x^{2}=3.79 ; p=\right.$ 0.05 ) and also increased with each BMI category. Strikingly, $41 \%$ of morbidly obese patients had resistant hypertension. The frequency of a CVD diagnosis was highest in the severely and morbidly obese.

Conclusions: Despite treatment with more anti-hypertensive agents, obese hypertensive patients had less effective blood pressure control. If lifestyle interventions and current treatment regimens are inadequate, new anti-obesity agents might be considered. A clinical trial of GLP-1 analogs in obese hypertensive patients with resistant hypertension is the logical next step.

Disclosures: None.

\section{Reference:}

1. Wilding JP, et al. Once-weekly semaglutide in adults with overweight or obesity. NEJM. 2021.

\section{P-26 Does CPAP affect blood pressure in hypertensive subjects with obstructive sleep apnea?}

\section{Sophie Hoskin ${ }^{2 *}$, Linda Mann ${ }^{1}$, lan Stone ${ }^{1}$, Abdul-Majeed Salmasi ${ }^{1}$}

${ }^{1}$ London North West University Healthcare NHS Trust, London, United Kingdom, ${ }^{2}$ Imperial College London, London, United Kingdom

Introduction: Obstructive sleep apnoea (OSA) is a known cause of hypertension. (1) This study looks at whether continuous positive airway pressure (CPAP) treatment for OSA has the ability to reduce blood pressure (BP).

Methods: We took systolic and diastolic BP measurements of patients who came to Central Middlesex Hospital to be given for CPAP treatment for OSA. Demographic details were also recorded. 3 months later, their subsequent blood pressure was recorded along with their compliance with the CPAP machine. Currently, data have been collected from 40 patients, with that number expected to increase over the coming months.

Results: $42 \%$ of patients diagnosed with OSA had previously diagnosed hypertension. After 3 months CPAP treatment, although there was a reduction in the mean systolic BP (SBP) decreased from 136.5 to $132.7 \mathrm{mmHg}(p=0.6010)$, and the mean diastolic BP (DBP) from 81.6 to $79.1 \mathrm{mmHg}$, this did not reach significance ( $p=0.6010$ and 0.1560 successively). The decrease was found to be the most profound in IndoAsian patients $(n=7)$, though did not reach significance where SBP decreased from 141.1 to $126.0 \mathrm{mmHg}(p=0.2108)$ and DBP decreased from 82.7 to $74.4 \mathrm{mmHg}(p=0.1428)$.

Conclusions: Although OSA is associated with raised BP, this preliminary study showed that CPAP treatment was not effective in reducing SBP and DBP in patients with OSA, especially those in the Indo-Asian population. Further similar studies are required with higher number of patients and using ambulatory BP monitoring.

Disclosures: The authors declared no competing interests.

Reference:

1. Dopp, J.M., Reichmuth, K.J. \& Morgan, B.J. Current Science Inc (2007) 9: 529. https://doi.org/10.1007/s11906-007-0095-2.

\section{P-27 Complications and treatment of hypercalciuria in familial hyperkalaemic hypertension (FHHt)}

\section{Abby Huckle ${ }^{1 *}$, Barian Mohidin ${ }^{1}$, Elizabeth Wan ${ }^{1}$, Keith Siew ${ }^{1}$, Stephen B Walsh'}

\section{${ }^{1}$ Department of Renal Medicine UCL, London, United Kingdom}

Introduction: Hypertension is frequently associated with hypercalciuria $^{1,2}$, nephrolithiasis ${ }^{3}$ and low bone mineral density ${ }^{4}$. Familial Hyperkalaemic Hypertension $(\mathrm{FHHt})$ causes hypercalciuria ${ }^{5}$, although complications of this are not reported.

Methods: We examined a cohort of 9 patients with genetically confirmed $\mathrm{FHHt}$. Biochemical, radiological, and clinical data was obtained in patients before and after thiazide treatment. All patients gave informed consent. The study had ethics committee approval (REC 05/Q0508/6). Data were compared using paired $t$ tests or Wilcoxon paired rank tests.

Results: 5 of the 9 patients were female (median age 41.7 years). The genetic diagnosis was confirmed in all patients, 5 patients had variants in KLHL3, 3 patients had variants of WNK4, and one had a variant of WNK1.

Pre-treatment potassium was high (median 5.6 IQR 5.2-6.2 $\mathrm{mmol} / \mathrm{L})$. Pre-treatment calcium was in the normal range (2.34 IQR 2.29-2.38 mmol/L). There was significant hypercalciuria with a raised urinary calcium/creatinine ratio (0.69 IQR 0.41-1.13). However, PTH (4 IQR 3.95-4.35 pmol/L), phosphate (1.15 IQR $1.25 \mathrm{mmol} / \mathrm{L}$ ) and alkaline phosphatase (57 IQR $45-84 \mathrm{mmol} / \mathrm{L}$ ) were all in the normal range.

Thiazide treatment significantly reduced hypercalciuria (calcium/creatinine ratio 0.15 IQR $0.05-0.29 p=0.04$ ) as well as the serum potassium (3.9 IQR 3.5-4.4 $\mathrm{mmol} / \mathrm{L} p=0.0167$ ).

Patients also developed complications of hypercalciuria. 3 patients had kidney stones demonstrated on cross-sectional imaging. One of these patients (male, 30 years old) had DXA criteria for osteoporosis ( $T$ score Femoral neck -1.5, lumbar spine -2.4).

Conclusions: This is the first case series to demonstrate complications of hypercalciuria (i.e., kidney stones) in patients with $\mathrm{FHHt}$. We demonstrate that thiazide treatment normalizes urinary calcium excretion. Thiazide treatment may have clinical utility in $\mathrm{FHHt}$ even if hypertension or hyperkalaemia are not problematic in order to avoid the complications of hypercalciuria. 
Disclosures: The authors have no relevant disclosures. References: None.

\section{P-28 Omitted}

\section{P-29 Omitted}

\section{P-30 Omitted}

\section{P-31 Omitted}

P-32 Sensitivity and reproducibility of IVC diameter and SVC flow velocity measurements to changes in cardiac pre-load in subjects with hypertension

\section{Ryan McNally ${ }^{1 *}$, Bushra Farukh ${ }^{1}$, Phil Chowienczyk ${ }^{1}$, Luca Faconti $^{1}$}

${ }^{1}$ King's College London, London, United Kingdom

Introduction: Measurements of the diameter of the inferior vena cava (IVC) and flow velocity in the superior vena cava (SVC) by cardiac ultrasound have been proposed as useful tools for assessing intra-vascular fluid volume and cardiac pre-load in acute settings $(1,2)$. Whether these measures can be used to guide fluid balance in primary hypertension is unknown. We evaluated the feasibility of using venae cavae-derived indices in detecting changes in cardiac pre-load within the physiological range in clinically euvolemic subjects with primary hypertension.

Methods: We investigated the sensitivity and reproducibility of inferior vena cava (IVC) diameters and superior vena cava (SVC) flow velocities in detecting changes in cardiac pre-load. These were obtained during passive leg raising (PLR) and lower limb venous occlusion (LVO), interventions which respectively transiently increase and decrease cardiac pre-load. Measurements were made in 36 clinically euvolemic subjects with hypertension and repeated on 2 occasions to examine reproducibility.

Results: During PLR, there was no significant change in IVC diameters, but peak flow velocity of the SVC $S$ wave increased by $6.5[95 \% \mathrm{Cl} 1.6-11.3] \mathrm{cm} / \mathrm{s}(P=0.01)$. During LVO, IVC diameter in expiration decreased by 3.2 [1.7-4.7] $\mathrm{mm}$ and the SVC S wave decreased by $9.7[4.4-14.7] \mathrm{cm} / \mathrm{s}(P<0.001)$. Bland-Altman plots showed good agreement between measurements of baseline IVC and SVC S wave measured on two separate days.

Conclusions: Venae cavae-derived indices can be used to assess changes in pre-load within the physiological range in primary hypertension and have potential for monitoring changes in cardiac pre-load.

\section{Disclosures: None.}

\section{References:}

1. Porter TR, Shillcutt SK, Adams MS, Desjardins G, Glas KE, Olson JJ, et al. Guidelines for the use of echocardiography as a monitor for therapeutic intervention in adults: A report from the American society of echocardiography. J Am Soc Echocardiogr. 2015;28(1):40-56.

2. Dipti A, Soucy Z, Surana A, Chandra S. Role of inferior vena cava diameter in assessment of volume status: a meta-analysis. Am J Emerg Med. 2012;30(8):1414-1419.e1. https://doi.org/ 10.1016/j.ajem.2011.10.017.
P-33 Transient cessation of deep brain stimulation triggers sympathetic activation in a case of severe resistant hypertension

Zoe H. Adams ${ }^{1 *}$, Angus K. Nightingale ${ }^{1,2}$, Richard P. Baker ${ }^{1,2}$, Stephen A. Klassen ${ }^{3}$, Kevin Shoemaker ${ }^{4}$, Julian F. R. Paton ${ }^{5}$, Emma C. Hart ${ }^{1}$, Nikunj K. Patel ${ }^{6}$

${ }^{1}$ CardioNomics Research Group, School of Physiology, Pharmacology and Neuroscience, University of Bristol, Bristol, United Kingdom, ${ }^{2}$ Bristol Heart Institute, University Hospitals Bristol and Weston NHS Foundation Trust, Bristol, United Kingdom, ${ }^{3}$ Department of Anesthesiology and Perioperative Medicine, Mayo Clinic, Rochester, USA, ${ }^{4}$ School of Kinesiology, Western University, London, Canada, ${ }^{5}$ Manaaki Mānawa, The Centre for Heart Research, University of Auckland, Auckland, New Zealand, ${ }^{6}$ Department of Neurosurgery, Southmead Hospital, North Bristol NHS Trust, Bristol, United Kingdom

Introduction: Deep brain stimulation (DBS) is an emerging treatment for severe, treatment-resistant hypertension. A 57-yearold man with severe uncontrolled hypertension $(>200 \mathrm{mmHg})$, history of ischemic heart disease, strokes and myocardial infarctions, gave written informed consent for surgical implantation of an electrode across the medial thalamus along a specific trajectory into the ventrolateral periaqueductal gray that delivers chronic intermittent DBS.

Methods: Fourteen-months post-implantation, haemodynamic responses to transient DBS cessation were recorded: muscle sympathetic nerve activity (MSNA, peroneal microneurography), continuous blood pressure (BP, Finometer) and heart rate (HR, ECG). Custom software detected MSNA action potentials (AP) (1). Cessation was tolerated for 2 minutes. Data were averaged every 20s. The last 20s of the OFF period and the first 20s of DBS resumption were compared to the minute preceding DBS cessation.

Results: Baseline BP (DBS ON) was 129/58 $\pm 7 / 3 \mathrm{mmHg}$ (mean \pm SD). Finometer BP was unreliable during DBS cessation. During the ON-OFF-ON protocol, HR increased from 57 to 66 and returned to 60 beats/min. MSNA increased from 42 to 54 and returned to 40 bursts/minute. AP incidence rose from 256 to 496 and returned to $338 \mathrm{AP} / 100$ heartbeats. APs/burst increased from 4.3 to 7.8 and returned to $3.9 \mathrm{AP} /$ burst. When APs were binned by peak-to-peak amplitude, DBS cessation showed activity in six of the largest amplitude bins, none of which were active at baseline, indicating recruitment of larger amplitude APs.

Conclusions: In this individual, cessation of DBS is associated acute and powerful sympathoactivation, detected by analysis of AP incidence and APs/burst. Furthermore, the activation of large, previously silent APs indicates recruitment of larger fibers (2). These data provide mechanistic insight into how DBS lowers blood pressure in resistant hypertension.

Disclosures: None.

References:

1. Salmanpour et al. 2010. J Neurosci Methods. 193(2):343-55.

2. Shoemaker JK. 2017. Clin Auton Res. 27(6):369-78.

P-34 Aortic pulse wave velocity is primarily determined by blood pressure but not other cardiovascular risk factors during childhood: a call to arms to standardize results

Karolis Azukaitis $^{1 *}$, Manish Sinha ${ }^{2}$, Augustina Jankauskiene, Bojko Bjelakovic ${ }^{3}$, Mieczysław Litwin ${ }^{4}$

${ }^{1}$ Clinic of Pediatrics, Institute of Clinical Medicine, Faculty of Medicine, Vilnius University, Vilnius, Lithuania, ${ }^{2}$ Kings College London Evelina London Childrens Hospital, London, UK, ${ }^{3}$ Clinic Of Pediatrics, Clinical 
Center, Nis, Serbia, Medical Faculty, University Of Nis, Nis, Serbia, ${ }^{4}$ Department Of Nephrology And Arterial Hypertension, The Childrens Memorial Health Institute, Warsaw, Poland

Introduction: Pulse wave velocity (PWV) is the gold-standard for non-invasive assessment of arterial stiffening in patients with hypertension. Adult studies have shown that PWV is mainly determined by blood pressure (BP) and age. We aimed to systematically analyze determinants of PWV in studies involving children with primary hypertension $(\mathrm{PH})$.

Methods: 196 articles retrieved from PubMed according to a predefined search strategy were screened for eligibility. 38 articles identified by title/abstract review underwent full-text screening by 2 reviewers and 12 were selected for the analysis if (i) measured carotid-femoral PWV (PWVcf); (ii) reported on risk associations using regression models; and (iii) included $\mathrm{BMI}$ and $\mathrm{BP}$ in the model.

Results: The total population included $>3700$ children with normal and elevated BP. 25\% studies used indirect methods for PWVcf estimation and $17 \%$ analyzed standardized PWVcf. Higher PWVcf in hypertensive vs non-hypertensive children was reported in $75 \%$ studies. $92 \%$ and $75 \%$ included age and sex, in regression models. In $83 \%$ studies PWV was positively associated with BP, whereas BMI associated with PWV in $25 \%$ only (positive 3 and negative association in 1). Age was positively associated in 18\% and no studies reported any significant associations with sex. Association of race were reported in 2 studies only (direct effect of non-white race in 1). Models including BP among other covariates (biochemical, anthropometric and other BP parameters) explained $12-88 \%$ of PWVcf variability (R2 not reported in $25 \%$ of studies).

Conclusions: $\mathrm{BP}$ is the predominate contributor to PWV, compared to the inconsistent or lesser contributions of BMI, age, sex or other CV risk factors during childhood. Additional studies are needed to determine whether increased PWV is a reflection of primary arterial disease or a secondary phenomenon. Our data also highlights the need of standardization and reporting of confounders, such as age and sex.

Disclosures: The abstract is based on the work developed by the COST Action HyperChildNET (CA19115) Working Group 3.

References: None.

\section{P-35 A hyperadrenergic state in primary hypertension associates with mood affective disorders and altered vascular responsiveness to centrally acting stimuli}

\section{Bushra Farukh ${ }^{1 *}$, Luca Faconti ${ }^{1}$, Ryan J McNally ${ }^{1}$, Phil Chowienczyk $^{1}$}

${ }^{1}$ King's College London, Ilford, United Kingdom

Introduction: A high prevalence of depression, anxiety and obstructive sleep apnoea (OSA) in primary hypertension may relate to chronic stress disrupting central GABAergic inhibitory neurons that regulate mood and autonomic activity. We investigated whether these stress sensitive co-morbidities may be dependent on basal sympathetic activity as measured by plasma normetadrenaline (NM) and whether vascular responses to centrally acting stimuli vary according to NM levels.

Methods: Subjects with primary hypertension ( $n=100,56 \%$ male) were stratified into tertiles of plasma NM concentration. Self-reported questionnaires included Patient Health Questionnaire-9 (PHQ-9) for depression, Generalized Anxiety Disorder-7 (GAD-7) for anxiety, and Epworth Sleepiness Score for OSA symptom severity. The forearm blood flow (FBF) response to mental stress elicited by the Stroop word-color test and that to device guided slow breathing (DGB) with breathing rate $<10$ breaths/min was measured.

Results: Subjects in the highest tertile of NM had greater BMI than those in the lowest tertile (mean \pm SEM) $(31 \pm 0.8$ vs $27 \pm$ $0.8 \mathrm{~kg} / \mathrm{m}^{2}, P<0.05$ ) but other characteristics including age and blood pressure were similar in these tertiles. Even after adjusting for $\mathrm{BMI}$, high NM individuals scored higher on depression (PHQ-9: $8.8 \pm$ 0.7 vs $4.6 \pm 0.7, P<0.01$ ), anxiety (GAD-7: $8.8 \pm 0.9$ vs $4.9 \pm 0.9, P<$ 0.05 ) and OSA questionnaires (ESS: $9.1 \pm 0.6$ vs $6.7 \pm 0.6, P<0.05$ ) compared to low NM subjects. Changes in BP were similar during mental stress and DGB but the FBF response to mental stress in high NM was impaired compared to subjects with low NM ( $28 \pm 3.8$ vs $47 \pm 5 \%$ increase above baseline, $P<0.05)$ and the response to DGB also differed in subjects with low compared to high NM $(P<0.05)$.

Conclusions: Greater plasma concentrations of normetadrenaline in primary hypertension identify patients with greater prevalence of depression, anxiety, OSA and altered vascular responsiveness to centrally acting stimuli.

Disclosures: None.

References: None.

\section{P-36 Vascular function in resistant and malignant hypertension}

\section{Reem Alsharari ${ }^{1,2,3^{*}}$, G Neil Thomas ${ }^{4}$, Gregory Lip ${ }^{3,5}$, Alena Shantsila ${ }^{3}$}

${ }^{1}$ Institute of Cardiovascular Sciences, University of Birmingham, Birmingham, United Kingdom, ${ }^{2}$ College of Applied Medical Sciences, King Saud Bin Abdulaziz University for Health Sciences, Riyadh, Kingdom of Saudi Arabia, ${ }^{3}$ Liverpool Centre for Cardiovascular Science, University of Liverpool and Liverpool Heart \& Chest Hospital, Liverpool, United Kingdom, ${ }^{4}$ Institute of Applied Health Research, University of Birmingham, Birmingham, United Kingdom, ${ }^{5}$ Aalborg Thrombosis Research Unit, Department of Clinical Medicine, Aalborg University, Aalborg, Denmark

Introduction: Increased carotid-femoral pulse wave velocity (cfPWV), high augmentation index (AIx), low subendocardial viability ratio (SEVR) and decreased flow-mediated dilation (FMD) have been associated with hypertension [1, 2]. The concomitant assessment of these parameters in patients with resistant hypertension $(\mathrm{RH})$ and malignant hypertension $(\mathrm{MHT})$ has been rarely studied.

Methods: In a cross-sectional comparison, 64 participants were recruited, 23 had $\mathrm{RH}(57 \pm 11$ years), $18 \mathrm{MHT}$ patients (54 \pm 13 years), and 23 normotensives controls (NC) (50 \pm 5 years). cfPWV, Alx and SEVR were assessed non-invasively. Endothelial function was assessed by FMD using a real-time edge detection technique.

Results: The groups were matched by age and body surface area $(p>0.05)$. Office and central blood pressures were elevated in MHT and RH groups compared to NC $(p<0.05)$. cf-PWV was increased in both hypertensives compared to NC $(p<0.05)$; however, no difference was observed in Alx between the groups ( $p=$ $0.20)$. SEVR was decreased in MHT compared to NC ( $p=0.01)$. FMD percentage in both hypertensives were markedly impaired (RH:5.45 $\pm 2.56 \%$ and MHT:5.97 $\pm 2.58 \%$ vs NC: $9.96 \pm 2.59 \%, p<$ $0.001)$. No differences in FMD percentage between MHT and $\mathrm{RH}$, $p=0.8$. Antihypertensive medications used were similar between $\mathrm{MHT}$ and $\mathrm{RH}$, except for the higher use of diuretics in $\mathrm{RH}$ group (100\% vs $67 \%)$. Using linear regression, independent predictors of high PWV were increased office systolic blood pressure and longer exposure to hypertension $\left(R^{2} 0.57 ; F 26.9 ; p<0.001\right)$.

Conclusions: Patients with $\mathrm{RH}$ and MHT appeared to have impaired endothelial function and increased arterial stiffness. 
Decreased SEVR in MHT may potentially imply that MHT has different features of abnormalities.

\section{Disclosures: None.}

\section{References:}

1. Panza et al. Abnormal endothelium-dependent vascular relaxation in patients with essential hypertension. $\mathrm{N}$ Engl J Med. 1990;323(1):22-7.

2. Kaess BM, Rong J, Larson MG, Hamburg NM, Vita JA, Levy D et al. Aortic stiffness, blood pressure progression, and incident hypertension. JAMA. 2012;308(9):875-81.

\section{P-37 Omitted}

\section{P-38 Dysregulation of central respiratory modulation of cardiac vagal tone is a novel, early sign of dysautonomia in patients with a-synucleinopathies}

\section{Carmela Maniero ${ }^{1,2^{*}}$, Peter Julu ${ }^{1,2}$, Mussadiq Shah ${ }^{2}$, Chris Wolff $^{2}$, Vikas Kapil ${ }^{1,2}$, Gurvinder Rull ${ }^{1,2}$, Ajay Gupta ${ }^{1,2}$, Manish Saxena $^{1,2}$, Paul Robinson ${ }^{1,2}$, Melvin Lobo ${ }^{1,2}$}

${ }^{1}$ Barts Health NHS trust, London, United Kingdom, ${ }^{2}$ William Harvey Research Institute and Barts NIHR Cardiovascular Biomedical Research Centre, Queen Mary University of London, London, United Kingdom

Introduction: Cardiac vagal tone (CVT) is the main central parasympathetic restraint of the heart controlling its chronotropic function. Central respiratory modulation of this chronotropic restraint is mediated by cholinergic interneurons connecting respiratory neurons to cardiac vagal motor neurons. In normal subjects the respiratory gating effects on CVT increases during deep breathing. These changes in respiratory gating of CVT are used to assess the integrity of cardiorespiratory neurons within the brainstem. We hypothesize that changes in the level of CVT during respiratory gating are early manifestations of central neurodegeneration.

Methods: Seven patients with complex circulatory disorders underwent autonomic function tests with the use Finapres beatto-beat BP monitoring and the NeuroScope (Medifit Instruments Ltd, London, UK). CVT was quantified continuously using the Linear Vagal Scale (LVS) in the supine position during regular and deep breathing at $0.1 \mathrm{~Hz}$ lasting for one minute.

Results: Four patients were male. Median age was 50.2 (IQ:54-59). Four presented with neurogenic orthostatic hypotension $(\mathrm{nOH})$, the others with BP variability or post-prandial hypotension. Three presented with additional neurological symptoms at the moment of the test. The others developed clinical manifestations of neurodegeneration on average 5.25 years later after the test.

Average baseline BP was $172 / 94 \mathrm{mmHg}$. Mean postural drop was $-34 /-14 \mathrm{mmHg}$. Average postural heart rate variation was 2 bpm, consistent with $\mathrm{nOH}$.

Resting supine CVT was abnormally low in all patients (1.81 \pm 0.6 LVS units; normal range 5-10 LVS Units). Five patients showed little or no respiratory modulation of CVT during deep breathing. The average CVT during deep breathing was $1.95 \pm 0.97$ LVS units with peaks at $3.67 \pm 2.55$ LVS units on average, normal range 5-15 LVS units).

Conclusion: Minimal or absent respiratory gating of CVT during deep breathing signifies marked impairment of cholinergic interneuron function in the lower brainstem and may be an early sign of central neurodegeneration.

Disclosures: None.

References: None.

P-39 Omitted
P-40 Learn With Nurses: delivering virtual clinical training to healthcare professionals around the world during the COVID19 pandemic

\section{Joanne Loades ${ }^{1 *}$, Michaela Nuttall ${ }^{1}$ \\ ${ }^{1}$ Learn With Nurses, London, United Kingdom}

Introduction: Learn With Nurses (LWN) is an initiative that offers free, dynamic and innovative training on blood pressure, cardiovascular risk and common long term conditions for healthcare teams around the world. Our webinars are developed and delivered by highly specialist practicing Registered Nurses who give their time free of charge, and while initially designed with nurses in mind, are open to all healthcare professionals (HPCs) and trainees.

LWN commenced training during the COVID-19 lockdown to help provide much needed education and support for HCPs through a very difficult time. Our aim is to help HCPs to answer the most frequently asked patient questions and provide knowledge of common health conditions that HCPs may need to understand and treat.

The COVID-19 pandemic has negatively affected cardiovascular disease prevention and evidence shows that the virus can be more serious for people who already have other health issues [1, 2]. Keeping HCPs up to date on topics such as atrial fibrillation, blood pressure, cholesterol, chronic kidney disease, diabetes, heart attacks and heart failure is vital to keeping people safe and wellunder "normal" times and especially now during COVID-19.

Our objectives are to:

Provide high quality, clinically driven education that is free and accessible to all HCPs around the world

- Actively engage with HCPs to promote an integrated approach to the delivery of care.

- Promote patients and their families as partners in decisions relating to their care.

- Promote current evidence based clinical practice guidance and signpost to credible resources.

- Contribute to improvements in the care of and quality of life of patients and their families.

- Support the health and wellbeing of HCPs.

Methods: Our Webinars are freely accessible to all healthcare professionals around the world. Days and times of the week vary to accommodate varying time zones and working patterns. Promotion is via social media and a dedicated webpage facilitates a simple bookings process. After booking participants receive a confirmation email with dial in facilities for the online delivery platform. Speakers log on from home to deliver the sessions and operate the system independently. Initially the platform allowed a maximum of 100 participants who are encouraged to interact using a chat function. Each webinar provides $30 \mathrm{~min}$ of taught content followed by $10 \mathrm{~min}$ remote discussion via a "Question and Answer" function.

On completion of the session participants receive a "pop up" electronic evaluation survey inviting them to score their knowledge of the subject before and after the session, their likelihood of using the knowledge they have gained and their trainer. They are also able to free-text comments and suggestions for further sessions. Results are automatically uploaded into feedback dashboards which are accessible for all to view via the webpage. A reminder is sent out $24 \mathrm{~h}$ following the session for any evaluations completed and participants receive a certificate of attendance and a PDF handout of key slides and information

Results: Since 18 May 2020 LWN has delivered a total of 158 webinars in all subjects. Registrations of 14,073 participants has yielded the attendance 7219 participants from 33 countries, an uptake of $51.3 \%$. 
For blood pressure sessions alone, the following has been achieved:

Number of sessions delivered: 23

Registrations: 2444

Attendances: 1233

Uptake: $50.5 \%$

Evaluation:

A total of 1029 responses have been received.

Ratings dashboard results (averages):

Knowledge of blood pressure before the webinar: 3.2 out of 5 Knowledge of blood pressure after the webinar: 4.4 out of 5

How likely are you to use what you have learned?: 4.7 out of 5

Please rate the Trainers delivery: 4.8 out of 5

Participants have cited the ease with which they could access our training (by phone, free, at home, no travel or childcare required); that it improved their confidence, competence, practice and knowledge; that they learned new things they shared with colleagues; and made them feel they could better explain these topics to patients. As one webinar attendee reported,

"[I] love how you can describe complicated subjects very simply."

In addition to feeling empowered, participants have also described feeling cared for, citing that they feel more valued, supported by connecting with others, and by having something to focus on. Perhaps our most poignant quote was the following:

"I don't want to go into details, but this has been keeping me going. At this time I can't afford to pay for training and this has been a lifesaver. I really appreciate it, stay safe:)"

Conclusions: LWN has identified a global unmet need for accessible clinical education during the COVID-19 pandemic. The sessions are brief and simply delivered, with clear explanations of common risk factors and conditions. In addition, feedback suggests that the sessions have had a positive impact on the wellbeing of healthcare professionals during a very challenging time.

Disclosures: None.

References:

1. Oxford Academic Health Science Network. CVD prevention during and after the COVID-19 pandemic: guidance for integrated care systems. 2020.

2. Primary Care Cardiovascular Society \& The British Heart Foundation. Influencing the redesign and recover of NHS health and care services for the detection and management of cardiovascular disease high risk conditions during the COVID-19 pandemic and beyond. 2020

\section{P-41 A shared decision-making tool for nurses and pharmacists for patients with diagnosed hypertensive patients in the UK and Ireland}

\section{Michaela Nuttall $^{1 *}$, Mark Cobain ${ }^{2}$, Joanne Loades ${ }^{3}$}

${ }^{1} \mathrm{C} 3$ Collaborating for Health, London, United Kingdom, ${ }^{2}$ Smart Health Solutions, London, United Kingdom, ${ }^{3}$ Younger Lives, London, United Kingdom

Introduction: Featuring the motivating CVD risk concept "Heart Age", patient perceptions of CVD risk and benefits of different intervention strategies. Patients and clinicians can explore different options for management framed, physical activity and medication, as a way of taking "years off" their Heart Age through blood pressure control. Heart Age has been shown to be effective in motivating risk factor reduction [1] and align perceived and measured risk [2].
Methods: We engaged with patients, nurses and pharmacists through established relationships within the community, third sector, charities, and social media. Using focus groups and surveys, we gained insight into hypertensive patient preferences for CVD risk framing, quantification of intervention benefits (and side effects) and processes to facilitate shared decision making. This knowledge underpins a training program and communities of practice.

Novel adaptations of the Heart Age tool was created to provide (years off) benefits for different aspects of blood pressure lowering, with a focus on the independent and joint benefits of medication management and lifestyle.

Clinicians using their skills and knowledge and the modified Heart Age, together with patients to arrive at joint decisions on blood pressure control with patients.

The pandemic created many challenges and the project became an entirely digital solution.

Results: This project will be completed by July 2021 and results for patients, nurses and pharmacists will be presented along with the challenges of delivering this project during a pandemic.

Conclusions: We believe that shared decision making can only be achieved if patients understand the benefit of personal cardiovascular risk and risk reduction to balance against other preferences.

Disclosures: Pfizer European Grant.

References:

1. Lopez-Gonzalez AA et al. Eur J Prev Cardiol. 2015;22 (3):389-96

2. Soureti A et al. Eur J Cardiovasc Prev and Rehab. 2009;17 (5):519-23

\section{P-42 Omitted}

\section{P-43 A point prevalence audit of undiagnosed and uncontrolled hypertension in a hospitalized patient cohort}

\author{
Kasie O' Reilly ${ }^{1 *}$, Paul Shiels ${ }^{2}$ \\ ${ }^{1}$ University of Limerick, Limerick, Ireland, ${ }^{2}$ Midlands Regional Hospital \\ Tullamore, Tullamore, Ireland
}

Introduction: There is a high correlation between cardiovascular disease (CVD) and mortality globally, which confers a significant burden on healthcare provision and resources [1]. Inadequate recognition and inappropriate management of CVD contribute to serious health complications and premature death [2]. This audit will evaluate the management of hypertension at Midlands Regional Hospital Tullamore (MRHT). Additionally, the data will assess the incidence of undiagnosed hypertension and the initiation of treatment in this group, if any, since admission.

Methods: A chart and bedside survey of all hospitalized inpatients over the age of 18 years. This audit defined uncontrolled hypertension as readings above $140 / 90 \mathrm{mmHg}$ (mean of last 3 readings on patient charts). This cut-off point also classified undiagnosed hypertension.

Results: The sample included 140 in-patients at MRHT. A total of $47 \%(n=66)$ of patients had consistently elevated blood pressure, $45 \%(n=30)$ of which had an existing diagnosis of hypertension. Overall, $26 \%(n=36)$ of patients were found to have undiagnosed hypertension, $64 \%(n=23)$ of which were not receiving any anti-hypertensive medications. $60 \%(n=44)$ of patients with diagnosed hypertension were controlled, with $43 \%$ ( $n=19$ ) receiving prescribed anti-hypertensive therapy. A majority with undiagnosed hypertension were not receiving antihypertensive therapy $(64 \%, n=23)$. 
Conclusions: This audit identified a delay in the diagnosis of hypertension and that satisfactory control of blood pressure is deficient. Enhanced vigilance in the hospital environment is recommended to limit the number of missed opportunities for treatment initiation, thus eradicating precipitating health consequences associated with cardiovascular disease.

Disclosures: None.

\section{References:}

[1] Murphy CM, et al. Hypertension prevalence, awareness, treatment and control in the over 50s in Ireland: evidence from the Irish longitudinal study on ageing. J Public Health. 2016 38(3), 450-58.

[2] Jones $\mathrm{N}$ et al. Diagnosis and management of hypertension in adults: NICE guideline update 2019. Br J Gen Pract. 2020;70 (691), 90-91.

\section{P-44 Urine analysis to assess patient adherence to hypertension medication (UPAH): a survey of general practitioners}

\section{Bethany Gilson ${ }^{1 *}$, Michael Burton ${ }^{1}$, Jannah Holmes ${ }^{1}$, Pankaj Gupta $^{2}$, Terry McCormack ${ }^{1}$}

${ }^{1}$ Hull York Medical School, Hull, United Kingdom, ${ }^{2}$ University Hospitals of Leicester, Leicester, United Kingdom

Introduction: Previous studies have shown that of those with resistant hypertension, $25 \%$ were not adhering to their treatment properly ${ }^{1}$. High pressure liquid chromatography can be used to test adherence of the forty most common antihypertensive medications, so can determine which patients actually have resistant hypertension and who are just non-adherent to medication. This project aimed to determine opinions of general practitioners around patient adherence and views on testing adherence to antihypertensive regimens.

Methods: A 6 question survey was developed, which enquired about knowledge of urine testing for anti-hypertensive adherence, as well as GP's views on testing adherence and any concerns they may have. This was sent to $40 \mathrm{GP}$ surgeries across the Hull York Medical School (HYMS) geographic footprint, with requests for GP's to complete the survey.

Results: 26 responses were received ( $0-4$ per surgery), of these, just one GP knew of the new research. $69 \%$ of participants believe extra antihypertensive medications are prescribed unnecessarily, and $54 \%$ think testing adherence would be useful. Most do not test drug adherence currently, with $13(50 \%)$ trusting patients to take medication after education of benefits, 3 not checking due to lack of available methods, 2 doing directly observed therapy and the rest monitoring indirectly by number of prescription requests. Whilst 14 (54\%) were keen on testing adherence, 46\% were not, most commonly due to concern about the doctor-patient relationship. Of the 54\%, 6 were concerned about impacting trust and the doctor-patient relationship.

Conclusions: About half of the GP's surveyed were willing to test adherence in this way, though the main concerns were impact on the doctor-patient relationship and trust. Therefore, this may have a place in primary care, though likely only for those who appear to have resistant hypertension.

Disclosures: None.

\section{Reference:}

1. Tomaszewski M. et al. Heart. 2014;100, 855-61.

P-45 The burden of "known" undiagnosed and untreated hypertension and why we will never solve this problem without appropriate investment in primary care

\section{James Boorer ${ }^{1 *}$}

\section{${ }^{1}$ Pathfields Medical Group, Plymouth, United Kingdom}

Introduction: High blood pressure is the second largest global risk factor for disease after poor diet [1]. Hypertension affects 1 in 4 adults in the UK and Public Health England estimate that for every 10 people diagnosed with hypertension in the UK a further 7 people have high blood pressure without a diagnosis or treatment [2]. We sought to assess the burden of diagnosed and undiagnosed hypertension across a primary care network in Plymouth with 30,194 patients and a population biased towards younger adults.

Methods: Database searches were created in the GP clinical system to identify patients with: Known hypertension; High blood pressure on last assessment without a hypertension diagnosis; In receipt of anti-hypertensives without a hypertension diagnosis.

Results: $12.5 \%$ of patients $(n=3780)$ had coded hypertension. A further $2 \%(n=590)$ did not have a hypertension diagnosis but were in receipt of anti-hypertensives. 9\% $(n=2725)$ had hypertension on their last BP without a diagnosis of hypertension: $8 \%$ $(n=2415)$ had stage 1 hypertension; $0.9 \%(n=280)$ had stage 2 hypertension; $0.1 \%(n=30)$ had stage 3 hypertension. We have run these searches with other practices and had similar results but do not have permission to quote these yet*.

Conclusions: The burden of known, undiagnosed hypertension is alarming when considering the potential for reducing avoidable morbidity, mortality and system cost through effective management of hypertension. Unfortunately there is little incentive for GP practices to proactively identify hypertension in their patients. NICE uses modeling costs of $£ 75 /$ patient/year for monitoring hypertension [3] whilst QOF remunerates practices at approximately $£ 5 /$ patient/year. Thus practices effectively loose resource if they try to detect hypertension and deliver optimal care. We believe it is time to fund hypertension detection and management appropriately to reduce avoidable morbidity, mortality and cost.

Disclosures: Lead Author is a principle share holder of Target Health Solutions Ltd that created the searches used to inform this study. Target Health Solutions is a software company that creates tools to assist in population health management.

*Prior to September I hope to provide near LCP wide statistics on this covering $100-150 \mathrm{~K}$ patients. I may have this data within the next 8 weeks. Of note we have already started to improve detection of hypertension and our stats were worse prior to this. Other practices where data has been seen have an even greater burden of undiagnosed hypertension. Furthermore if the BIHS have any specific population health questions regarding hypertension we could probably create suitable searches and present these as well.

\section{References:}

[1] Global, regional, and national comparative risk assessment of 79 behavioral, environmental and occupational, and metabolic risks or clusters of risks in 188 countries, 1990-2013: a systematic analysis for the Global Burden of Disease Study 2013. GBD 2013 Risk Factors Collaborators. Lancet. 10/9/2015. https://doi.org/ 10.1016/S0140-6736(15)00128-2.

[2] Health matters: combating high blood pressure, Public Health England, 2017. https://www.gov.uk/government/ publications/health-matters-combating-high-blood-pressure/ health-matters-combating-high-blood-pressure.

[3] Hypertension in adults: diagnosis and management. Costeffectiveness analysis: treatment initiation threshold for people with stage 1 hypertension. NICE guideline NG136. Economic analysis report. 2019. https://www.nice.org.uk/guidance/ng136/ evidence/costeffectiveness-analysis-treatment-initiationthreshold-for-people-with-stage-1-hypertension-pdf-6957345277. 
P-46 Urine analysis to assess patient adherence to hypertension medication (UPAH): A survey of secondary care physicians

\section{Jannah Holmes ${ }^{1 *}$, Bethany Gilson ${ }^{1}$, Michael Burton ${ }^{1}$, Pankaj Gupta $^{2}$, Terry McCormack ${ }^{1}$}

${ }^{1}$ Hull York Medical School, Hull, United Kingdom, ${ }^{2}$ University Hospitals of Leicester, Leicester, United Kingdom

Introduction: $25 \%$ of patients on anti-hypertensive medication are totally or partially non-adherent ${ }^{1}$. The use of urine spot tests and liquid chromatography-tandem mass spectrometry-based biochemical analysis (LC-MS/MS) can test adherence of the forty most prescribed antihypertensive medications within the last 6 days. The aim of this project was to explore the insights of secondary care physicians into adherence and ascertain if there is a use for urine spot tests in the Hull York Medical School (HYMS) area.

Methods: A 6-question qualitative survey was sent to elderly care physicians, cardiologists, and renal specialists within the HYMS region $(n=63)$. The survey focused on knowledge of the new technique, whether it would be useful in this region and reservations around using LC-MS/MS.

Results: We obtained 10 responses (response rate $15.8 \%$ ). 50\% of participants were not aware of the current research, 1 practitioner was up to date. $90 \%$ believed patients are prescribed extra antihypertensives that are not needed. $80 \%$ wanted to test patients on multi-hypertensives who show resistance, whilst $20 \%$ would test all patients with hypertension. $40 \%$ of physicians do not currently test for adherence due to lack of a method and $60 \%$ have used techniques such as direct observational therapy. LC-MS/MS testing is offered by South Tees Hospital Trust, but samples are sent to Leicester for processing, delaying the results. $70 \%$ would use this technique if it were shown to improve adherence and/or became available in the local biochemical department.

Conclusions: This questionnaire has shown that most physicians would be willing to use the LC-MS/MS method to test antihypertensive adherence in their patients. The main reservations highlighted were the damage to the doctor patient relationship and the Hawthorne effect. Both reservations could potentially be overcome with the possibility of post-test consent.

Disclosures: None.

Reference:

1. Tomaszewski M. et al. Heart 2014;100:855-61.

P-47 Urine analysis to assess patient adherence to hypertension medication (UPAH): A survey of chemical pathology departments

\section{Michael Burton ${ }^{1 *}$, Jannah Holmes ${ }^{1}$, Bethany Gilson ${ }^{1}$, Pankaj} Gupta $^{2}$, Terry McCormack ${ }^{1}$

${ }^{1}$ Hull York Medical School, Hull, United Kingdom, ${ }^{2}$ University Hospitals of Leicester, Leicester, United Kingdom

Introduction: The benefits of vigorous antihypertensive control are well demonstrated and yet many patients do not to adhere to their medication [1]. Recent research has demonstrated screening for antihypertensive adherence in patients' urine samples with high-performance chromatography-tandem mass spectrometry (HPLC-MS/MS) leads to improved outcomes and compliance [2]. Little research has been conducted into the attitudes of chemical pathology departments towards this investigation, to understand the barriers that may prevent its implementation.

Methods: We developed a 6 question qualitative survey to assess the attitudes of chemical pathology departments towards the use of HPLC-MS/MS for antihypertensive compliance. The study was targeted toward the 4 chemical pathology departments found within the Hull York Medical School geographical footprint.

Results: All departments responded. $100 \%$ of departments supported the use of the investigation, but only when there is clinical suspicion of non-compliance. Departments were unwilling to invest in the equipment locally, due to high cost and lack of demand, but supported centralizing the testing regionally or nationally. $50 \%$ of departments currently use the services offered by Leicester and reported high satisfaction. Suggested barriers to the investigation included; turnaround time, cost, undermining the doctor-patient relationship and technical complexity.

Conclusions: The results of this survey suggest there is a place for antihypertensive adherence monitoring with HPLC-MS/MS, although further investigation and/or education is required with regards to the barriers that may prevent its implementation.

Disclosures: No declaration of interest or funding. University Ethics Approval: 20-36 09 Jul 2020.

Keywords:

Urine analysis, Adherence, Chemical Pathology Department

References:

1. Collins R et al. Blood pressure, stroke, and coronary heart disease: part 2, short-term reductions in blood pressure: overview of randomized drug trials in their epidemiological context. Lancet. 1990;335(8693):827-38.

2. Gupta $P$ et al. Biochemical screening for nonadherence is associated with blood pressure reduction and improvement in adherence. Hypertension. 2017;70(5):1042-48. 